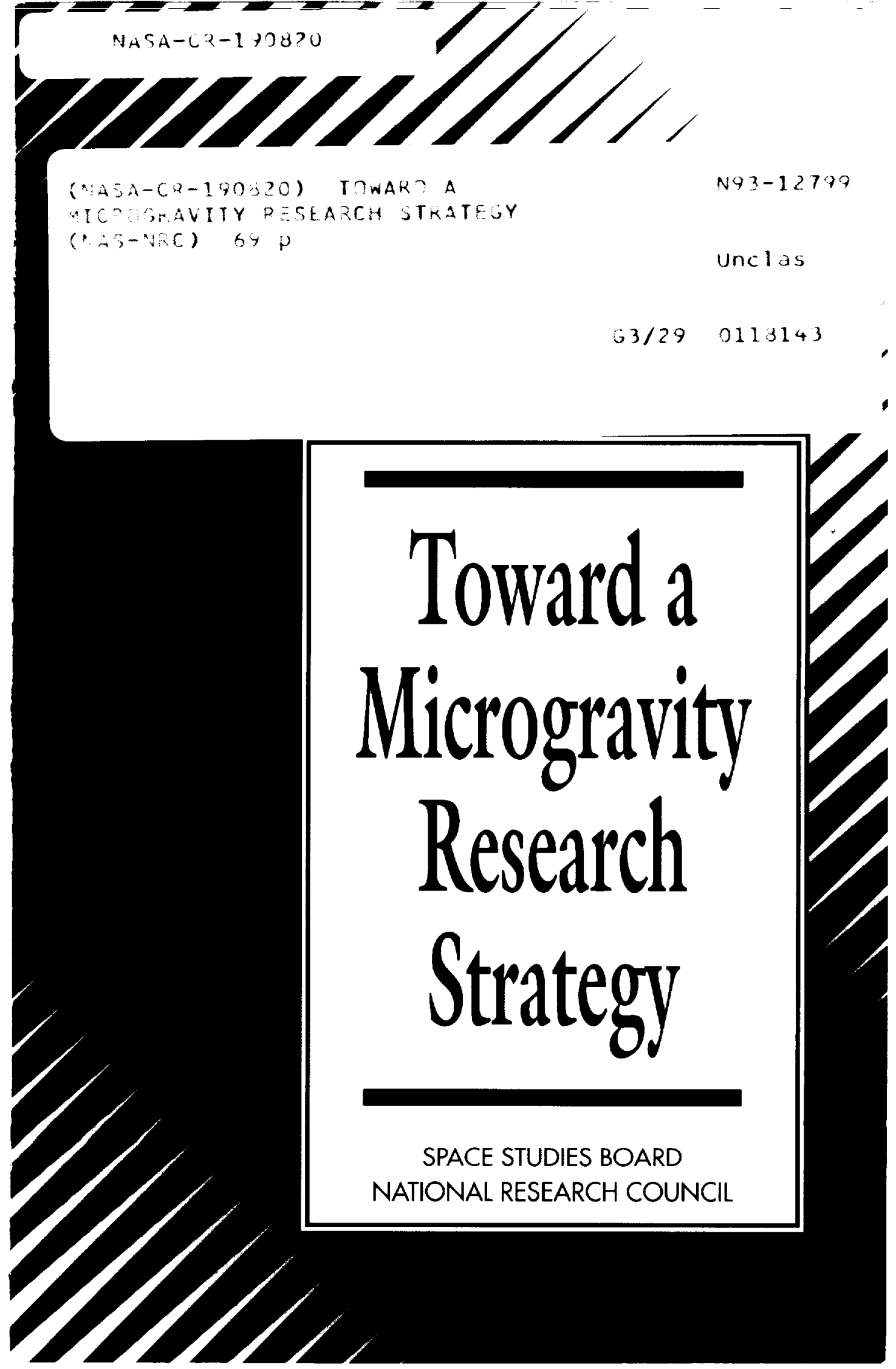




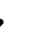




\section{Toward a Microgravity Research Strategy}

Committee on Microgravity Research

Space Studies Board

Commission on Physical Sciences, Mathematics, and Applications

National Research Council

NATIONAL ACADEMY PRESS

Washington, D.C. 1992 
NOTICE: The project that is the subject of this report was approved by the Governing Board Council, whose members are drawn from the councils of the National of the National Research Councion Academy of Sciences, the National Academ their special competences The members of the committee responsible

and with regard for appropriate balance.

This report has been reviewed by a group other than the authors actional Academy of approved by a Report Review Committee consisting of members of the National

Sciences, the National Academy of Engineering, ar

The National Academy of Sciences is a private, nonprofit. self-perpetuating society of distinguished scholars engaged in scientific and engineering research, dedicated to the furtherance f science and technology and to their use for the general welfare. Upon the authority of the charter granted to it by the Congress in 1863, the Academy has a mandate that requires it to dise federal government on scientific and technical matters. Dr. Frank Press is president The National Academy of Engineering was established in 1964, under the chareng of outstanding engineers. It is autonomous in its administration and in the selection of its members, sharing with the National Academy of Sciences the responsibility for advising the federal government. The National Academy of Engineering also sponsors engineering programs aimed at meeting national mineers. encourages education and research, and recognizes the superior achievemering.

Dr. Robert M. White is president of the National Academy of Engineering Academy of Sciences to

The Institute of Medicine was established in 1970 by the Nation in the examination of policy secure the services of eminent members of appropriate professions in the responsibility given matters pertaining to the health of the public. The Institute acts under the respon adviser to the to the National Academy of Sciences by its congressionify issues of medical care, research. federal government and, upon its own initiative, to identify issues of medicine.

and education. Dr. Kenneth I. Shine is president of the Institute of Mcademy of Sciences in 1916

The National Research Council was organized by the National Academy of Sciences to associate the broad community of science and technology with the Academs in accordance with furthering knowledge and advising the federal government. Funcipal operating general policies determined by the Academy, the Council has becol Academy of Engineering in agency of both the National Academy of Sciences and the National Academentic and engineering communiproviding services to the government, the public, and the scient the Institute of Medicine. Dr. ties. The Council is administered jointly by both Academies and the Institute of Frank Press and Dr. Robert

National Research Council.

Support for this project was provided by Contract NASW 4627 between the National Academy of Sciences and the National Aeronautics and Space Administration.

Copies of this report are available from

Space Studies Board

National Research Council

2101 Constitution Avenue, N.W.

Washington, D.C. 20418

Printed in the United States of America 


\section{COMMITTEE ON MICROGRAVITY RESEARCH}

ROBERT F. SEKERKA,* Carnegie Mellon University, Chairman ROBERT A. BROWN, Massachusetts Institute of Technology FRANKLIN D. LEMKEY, United Technologies Research Center WILLIAM A. SIRIGNANO, University of California, Irvine THOMAS A. STEITZ, The Howard Hughes Medical Institute

Space Studies Board Member (July 1989 to June 1991) JOHN R. CARRUTHERS

Space Studies Board Staff

JOYCE M. PURCELL, Executive Secretary MELANIE M. GREEN, Administrative Secretary CARMELA J. CHAMBERLAIN, Administrative Secretary 


\section{SPACE STUDIES BOARD}

LOUIS J. LANZEROTTI, AT\&T Bell Laboratories, Chairman

JOSEPH A. BURNS, Cornell University

ANDREA K. DUPREE, Harvard-Smithsonian Center for Astrophysics

JOHN A. DUTTON, Pennsylvania State University

LARRY W. ESPOSITO, University of Colorado, Boulder

JAMES P. FERRIS, Rensselaer Polytechnic Institute

HERBERT FRIEDMAN, Naval Research Laboratory (retired)

RICHARD L. GARWIN, IBM T.J. Watson Research Center

RICCARDO GIACCONI, Space Telescope Science Institute

NOEL W. HINNERS, Martin Marietta Civil Space and Communications

Company

JAMES R. HOUCK, Cornell University

DAVID A. LANDGREBE, Purdue University

RICHARD S. LINDZEN, Massachusetts Institute of Technology

JOHN H. MCELROY, University of Texas at Arlington

WILLIAM J. MERRELL, JR., Texas A\&M University

RICHARD K. MOORE, University of Kansas

ROBERT H. MOSER, The NutraSweet Company

NORMAN F. NESS, University of Delaware

MARCIA NEUGEBAUER, Jet Propulsion Laboratory

MARK SETTLE, ARCO Oil and Gas Company

WILLIAM A. SIRIGNANO, University of California, Irvine

FRED TUREK, Northwestern University

ARTHUR B.C. WALKER, Stanford University

MARC S. ALLEN, Director 


\section{COMMISSION ON PHYSICAL SCIENCES, MATHEMATICS, AND APPLICATIONS}

NORMAN HACKERMAN, Robert A. Welch Foundation, Chairman PETER J. BICKEL, University of California at Berkeley GEORGE F. CARRIER, Harvard University GEORGE W. CLARK, Massachusetts Institute of Technology DEAN E. EASTMAN, IBM T.J. Watson Research Center MARYE ANNE FOX, University of Texas PHILLIP A. GRIFFITHS, Institute for Advanced Studies NEAL F. LANE, Rice University ROBERT W. LUCKY, AT\&T Bell Laboratories CLAIRE E. MAX, Lawrence Livermore Laboratory CHRISTOPHER F. MCKEE, University of California at Berkeley JAMES W. MITCHELL, AT\&T Bell Laboratories

RICHARD S. NICHOLSON, American Association for the Advancement of Science ALAN SCHRIESHEIM, Argonne National Laboratory KENNETH G. WILSON, Ohio State University

NORMAN METZGER, Executive Director 



\section{Contents}

SUMMARY AND RECOMMENDATIONS 1

Introduction, 1

Nature of the Field, 2

Status of the Field, 2

The Conduct of Microgravity Research, 3

Conclusions and Recommendations, 4

References, 6

1 OVERVIEW OF MICROGRAVITY RESEARCH

Examples of Microgravity Experiments, 8

Reference, 10

2 STATUS OF THE FIELD

References, 13

3 THE CONDUCT OF MICROGRAVITY RESEARCH

Instrumentation, 14

Manned Versus Robotic Interaction, 15

Range of Microgravity Facilities, 16

Microgravity Research Outside the United States, 18

Commercial Programs, 19

The Research and Analysis Program, 21

References, 22

PRECEDING PAGE BLANK NOT FILMED

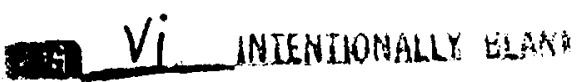


4 TOWARD THE DEVELOPMENT OF A RESEARCH STRATEGY

APPENDIXES

A Biological Sciences, 29

B Combustion Science, 35

C Electronic Materials, 40

D Fluids, Interfaces, and Transport, 45

E Glasses and Ceramics, 52

F Metals and Alloys, 56 


\section{Summary and Recommendations}

\section{INTRODUCTION}

As part of a self-assessment and subsequent reorganization in 1989 , the Space Studies Board (SSB) created a new standing committee-the Committee on Microgravity Research (CMGR). The formation of the committee was due, in part, to the dissolution of the National Research Council's (NRC's) Space Applications Board, which, until 1988, held the NRC's advisory responsibility for microgravity issues.

Over the course of the past 20 years, the Space Studies Board has, through its standing discipline committees, developed and published a series of research strategies for each of the major space research disciplines. These strategies are meant to serve as guides for the National Aeronautics and Space Administration (NASA) in planning its space research program. As one of its charges, the CMGR was asked by the SSB ". . . to conduct a study on the maturity and state of readiness of the field for the development of a comprehensive long-range research strategy."

In this report to the SSB, the CMGR finds that the various subdisciplines of the field are heterogeneous in both their nature and state of maturity. This is reflected in Appendixes A to F, which briefly discuss the status, accomplishments, and prospects and opportunities for each microgravity research subdiscipline. Notwithstanding this inherent heterogeneity, the CMGR concludes that the field as a whole would benefit from the formulation of a long-range research strategy and that such a strategy should be developed as soon as possible. 


\section{NATURE OF THE FIELD}

Microgravity research encompasses scientific investigation conducted in a gravitational field (or equivalent acceleration with respect to an inertial frame) that is a small fraction of the gravitational acceleration on Earth. The role of gravity in physical phenomena is uniquely important in a limited set of circumstances, including the following:

1. As a driving force for convection in fluids,

2. As a driving force for phase separation,

3. As a force that helps to determine the free surface morphology of fluids,

4. Near a critical point,

5. In the presence of very weak binding forces,

6. In the presence of very large masses or for very long times, and

7. In structural members or over large distances.

To date, most microgravity experiments have been focused on exploring the first two roles above. These experiments have included studies of crystal growth in fluids, fundamental phenomena in crystal growth, convection phenomena, measurement of the transport properties of fluids, combustion phenomena, fire safety aboard spacecraft, and immiscible alloys and multiphase solids.

\section{STATUS OF THE FIELD}

Between 1989 and 1991, the CMGR reviewed the status of microgravity research, the activities of NASA's Microgravity Science and Applications Division, and previous studies such as Materials Processing in Space, ${ }^{1}$ Microgravity Science and Applications, ${ }^{2}$ Review of Microgravity Science and Applications Flight Programs-January-March 1987, ${ }^{3}$ and Fluid Sciences and Materials Science in Space-a European Perspective. ${ }^{4}$ Based on this review, the CMGR reached the following conclusions.

Fluids, interfaces, and transport; metals and alloys; and combustion science are more developed than the other subdisciplines of the field. The biological sciences category shows promise in the area of protein crystal growth, but little in other aspects such as electrophoresis. Current research holds out little hope for explaining why protein crystals grow differently in space or how to exploit the differences. Excellent-although only a fewexperiments are planned in the subdiscipline of fundamental processes. Recommendations for future experiments in this direction are more likely to be derived from unsolicited proposals than from Announcements of Opportunity (AOs) issued by NASA. Research in the area of electronic materials has 
concentrated on bulk materials thus far. There is some indication that these experiments will produce information of scientific importance. However, concentration on bulk materials is contrary to the mainstream of the field, which emphasizes research on the properties of thin films deposited on substrates rather than research on electronic properties. Current research on the qualities of bulk crystals (when used as substrates) suggests that such crystals may hold some practical value. The subdiscipline of glasses and ceramics is relatively undeveloped at present; some research in this area overlaps with research in metals and alloys.

It should be recognized that microgravity research is a relatively new and laboratory-intensive field that requires frequent access to space. So far, progress has been limited considerably by the paucity of flight opportunities.

\section{THE CONDUCT OF MICROGRAVITY RESEARCH}

Microgravity research must be performed in an environment far from Earth and, therefore, is largely inaccessible. In addition, it is extremely expensive, both in terms of the initial investment and in operating costs, particularly when humans are involved.

The conduct of microgravity research requires the development of scientific equipment that is capable of withstanding the stresses of launch and reentry and of functioning reliably and safely in space. The interaction of users with this equipment is quite different from their interaction with other space instruments. Often, the users of microgravity equipment must change experimental parameters from run to run of an experiment. A more efficient approach to designing and building equipment would be to provide instrumentation that is specific to the experiment or class of experiments and that is designed and built in close cooperation with the principal investigator(s). This would be a departure from current practice, in which equipment is developed for a broad population of users.

Microgravity experiments can be carried out in a variety of modes, ranging from continuous human intervention to full automation. An optimum microgravity research program would use a mixture of modes, depending on the set of experiments to be performed, the state of the technology, and cost-effectiveness. Some microgravity experiments require a manned, space-based laboratory (such as a space station), while others can be done well or better, and at a much lower cost, by other means such as in satellites, rockets, and drop towers.

A wide range of facilities-from ground-based drop tubes to the complex facilities of the Shuttle-based Spacelab-can provide microgravity conditions. An experimenter's choice of facility should be based on specific research needs as well as cost. 


\section{CONCLUSIONS AND RECOMMENDATIONS}

\section{Development of a Research Strategy}

The Committee on Microgravity Research recommends that a longterm research strategy, such as that developed by the Space Studies Board's other discipline committees, be developed for microgravity science. In addition to defining the overall goals of the microgravity science field and summarizing the current knowledge of its subdisciplines, this strategy should identify the fundamental questions that need to be addressed and the scientific community's ability to address them. Consideration should be given to all modes of doing this type of research, with attention to maximizing experimental return and minimizing cost. The primary objectives defined should be ranked in order of priority and should be accompanied by the criteria used to determine their priority. Critical components necessary to support a successful microgravity research program should be described and appropriate measurement indicators developed.

\section{Microgravity Research Versus Materials Processing}

It should be recognized that, to date, no examples have been found of materials that are worthy of manufacture in space. Unless and until such examples are found, space manufacturing of products to be used on Earth should be deemphasized as a reason for undertaking microgravity research. The descriptor "materials processing" is misleading and should be eliminated. The CMGR recommends that "microgravity research" be used instead. The main rationale for the microgravity research program should be to improve our fundamental scientific and technological knowledge base, particularly in areas that are likely to lead to improvements in processing and manufacturing on Earth. A secondary rationale should be to develop the technologies for handling materials in space and possibly for processing materials to be used in space.

\section{Subdivisions for Microgravity Research}

Microgravity research encompasses a wide range of subdisciplines. NASA's Microgravity Science and Applications Division and its advisory groups are currently divided into seven "disciplines": biological sciences; combustion science; electronic materials; fluids, interfaces, and transport; fundamental processes; glasses and ceramics; and metals and alloys.

After careful consideration, the Committee on Microgravity Research has concluded that the current subdivisions of microgravity science should be revised. The CMGR recommends that microgravity research be re- 
organized into six categories that reflect future opportunities more realistically, including:

- Biological science and technology,

- Combustion,

- Fluid science,

- Fundamental phenomena,

- Materials, and

- Processing science and technology.

\section{Conduct and Support of the Research and Analysis Program}

A thorough program of ground-based research should precede and follow every microgravity flight. When exemplary materials are produced in microgravity, attempts should be made to replicate them using ground-based research. In addition, much more effort should be made to model phenomena suggested by microgravity observations.

Research projects include both focused opportunities advertised through AOs issued by NASA and unsolicited proposals submitted to NASA. The research and analysis program in NASA's Microgravity Science and Applications Division consists of the ground-based research needed to provide the context of knowledge from which the flight program originates as well as the infrastructure required to analyze microgravity experiments in a broader context. If microgravity research is to develop into a mature field, the current research program should be reconstituted and refocused in order to improve its health and to provide new opportunities. The CMGR recom. mends that NASA apply a set of value criteria and measurement indicators to define the research and analysis program more clearly. These cal and chemiteria and indicators should be compared with other areas of physi-

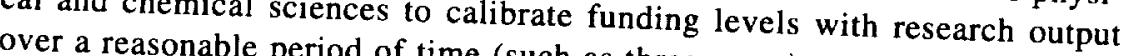

If research period of time (such as three years).

into the microgravigher quality and wider diversity is to be incorporated and analysis budget besearch program, it is imperative that the research The CMGR recommends larger fraction of the total microgravity budget. sis in microgravity science be funding level for research and analytotal program of NASA's Microgravity Science and percentage of the sion in order to build a strong scientific base for and Applications Divi-

\section{Content of the Program and Facilities}

Materials employed in microgravity environments should be characterized thoroughly before and after flight. The thermophysical data needed to 
interpret experiments should be measured as a part of the program if they are not available in the literature. Contemporary interest in electronic materials focuses on thin films. Bulk electronic materials are of secondary importance and should be studied in microgravity only to the extent that they will yield fundamental knowledge about processing.

When promising results have been obtained, experiments should be repeated to examine their reproducibility; in particular, experiments should be designed and conducted to learn why microgravity makes a measurable difference. Experiments should be analyzed and classified according to their minimum facility requirements so that they can be carried out in the most cost-effective manner. The committee recommends that a concerted effort also be made to classify experiments according to their minimum needs in order that the most cost-effective access to reduced gravity will be used. Equipment to accomplish specific experiments should be designed and built in close cooperation with the principal investigator(s). The acceleration vector environment must be measured accurately, locally, frequently, and synchronously with every experiment. These data should be provided to the principal investigators immediately. Whenever exemplary materials are produced in microgravity, considerable effort should be exerted to replicate them in ground-based research.

\section{Commercial Programs}

In addition to the activities financed by NASA's Microgravity Science and Applications Division, NASA funds commercial microgravity research through its Office of Commercial Programs. This office provides incentives for space experiments and, in cooperation with industry, has established centers for the commercial development of space (CCDS) at several universities. Started in 1986, these centers were given five years in which to become independent through increased industrial funding. The CMGR recommends that a thorough technical review of the centers for commercial development of space be conducted to determine the quality of their activities and to ascertain to what degree their original mission has been accomplished.

\section{REFERENCES}

1. Committee on Scientific and Technological Aspects of Materials Processing in Space, Acations Board 1978. Materials Processing in Space. National Academy of Sciences, Washington, D.C.

. Microgravity

2. Solid State Sciences Committee, Board on Physics and Acrodemy Press, Washington, D.C. Science and Applications: Report on a Workshop. National Academy Press, Wicrogravity Sci

3. Review Committee, J. Robert Schrieffer, chairman. 1987. Reviewsities Space Research ence and Applications Flight Prog

Association, Washington, D.C. 1987. Fluid Sciences and Materials Science in Space-a

4. European Space Agency. 1987. 


\section{Overview of Microgravity Research}

Microgravity research is scientific investigation conducted in a gravitational field (or equivalent acceleration with respect to an inertial frame) that is a small fraction of the gravitational acceleration, $g_{\mathrm{E}}$, on Earth. Strictly speaking, the prefix "micro" would imply a field of $10^{-6} g_{E}$, but the word "microgravity" is used in a more generic sense in this report to describe gravitational fields that are typically less than $10^{-2} g_{\mathrm{E}}$ and might be lower than $10^{-6} g_{\mathrm{E}}$. In consideration of future missions to the Moon and to other planets, there might also be interest in studies at other levels of reduced gravity, for example, $0.16 g_{\mathrm{E}}$ (Moon) or $0.3 g_{\mathrm{E}}$ (Mars).

Gravity is certainly a weak force compared to the "strong" or "weak" nuclear forces that bind atomic nuclei or subnuclear particles or to the electromagnetic forces that bind atoms and molecules. Therefore, the role of gravity in physical phenomena is important only when stronger forces are already in balance or when other special circumstances arise. Consequently, gravity is important in the following cases:

1. As a driving force for convection in fluids. Differences in density, resulting from inhomogeneity in temperature and/or composition, can cause an otherwise quiescent fluid to convect, thus giving rise to convective heat and mass transport. In fluids, on Earth, convection of molecular species is typically orders of magnitude more rapid than the slow migration caused by molecular diffusion due to Brownian motion.

2. As a driving force for phase separation. Once thermodynamic considerations (the equalization of chemical potentials dominated by elec- 
tromagnetic forces) have led to coexisting phases, such phases still can have different densities, and phase separation can take place, even by sedimentation over long periods of time, if the difference in density is slight.

3. As a force that helps to determine the free surface morphology of fluids. Even at or near thermodynamic equilibrium, single phases or multiphase systems will be bounded by surfaces or interfaces whose morphology is determined by a balance of forces, including gravitational forces. Examples are the shapes of bubbles, droplets, and liquid zones.

4. Near a critical point. Near such a point, the balance of forces is so delicate that thermodynamic and transport phenomena can exhibit divergent or otherwise anomalous behavior. Thus even the slight inhomogeneity in hydrostatic pressure that arises whenever matter is in a gravitational field can lead to important differences in observable features.

5. In the presence of very weak binding forces. There could be cases, particularly in living systems, in which gravitational forces play a subtle but important role, possibly because of the weak forces that bind macromolecules. Examples might be the degree of perfection of a crystallized protein or the development of a cell, an embryo, or a plant.

6 . In the presence of very large masses or for very long times. Examples are relativistic phenomena such as the bending of starlight and gravitational waves.

7. In structural members or over large distances. Examples include stresses in buildings and bridges or phenomena within Earth and its atmosphere.

\section{EXAMPLES OF MICROGRAVITY EXPERIMENTS}

Most microgravity experiments and applications to date have involved gravity as a driving force for (1) convection in fluids and (2) phase separation. Examples of such experiments include the following:

\section{For gravity as a driving force for convection in fluids}

a. Crystal growth from fluids. The growth of crystals from either the melt, vapor, or solution can be quite different in microgravity conditions than on Earth because of reduced convection. Sometimes reduced convection can create a product that has a more uniform composition and structure but is not necessarily a better one from the standpoint of applications. Moreover, the virtual absence of convection results in a process that is controlled by molecular diffusion and, therefore, is usually more predictable and amenable to modeling.

b. Fundamental phenomena in crystal growth. The fundamental mechanisms governing phenomena that occur during crystal growth, 
particularly those that pertain to crystal-fluid interface morphology (dendrites, cells, and the structure of in situ eutectic composites), are not well understood and often are masked by convection that is unavoidable on Earth. Carefully designed experiments conducted in microgravity might guide choices among alternative theories.

c. Convection phenomena. In microgravity, buoyancy forces in the equations of fluid dynamics can be negligible, or at least adjustable, thus allowing the study of other phenomena. Examples are convection driven by secondary mechanisms, such as surface tension gradients (sometimes called Marangoni convection), and turbulence that is dominated by inertial and viscous forces.

d. Measurement of the transport properties of fluids. The transport properties of fluids, characterized by such features as diffusion coefficients and Soret coefficients (diffusion driven by thermal gradients), can be measured much more accurately in microgravity experiments, leading to data with far less scatter than data obtained on Earth (sometimes even to transport coefficients with signs opposite to those measured on Earth) and to the possibility of identifying controlling mechanisms.

e. Combustion phenomena. Typically, buoyancy-driven convection plays a major role in the transport of materials associated with combustion processes that occur over large lengths and/or at small velocities. The very notion of a flame invites the image of rapidly rising incandescent gases. However, flammability and flame propagation are quite different in microgravity than on Earth. It should be possible to conduct experiments with nearly spherical symmetry that would permit comparison of the results with tractable models.

f. Fire safety aboard spacecraft. From the perspective of fire safety aboard spacecraft, it is important to understand and control the practical consequences associated with combustion phenomena.

2. For gravity as a driving force for phase separation

a. Immiscible alloys and multiphase solids. Phase separation resulting from sedimentation can be avoided in microgravity, thus possibly giving rise to uniformly dispersed composite materials. However, uniform dispersal will be possible only if other phenomena (e.g., Brownian motion and Marangoni convection) can be overcome. Furthermore, two-phase fluid flow can be studied with attention to such characteristics as "slug flow," which can influence heat transfer coefficients.

Among the experiments based on other roles of gravity are the following: 
3. For gravity as a force that helps to determine the free surface morphology of fluids

a. Containerless processing. Given the virtual weightlessness of materials in microgravity, it should be possible to process highly reactive or ultrapure materials by avoiding contact with a container, although some slight force is necessary to maintain positional stability.

b. Drop dynamics. The dynamics (e.g., vibrational modes and bifurcations) of freely floating droplets can be studied experimentally and compared with theoretical models to enhance our understanding of nonlinear continuum mechanics.

\section{For gravity near a critical point}

a. The Lambda Point Experiment, whose objectives are to measure the heat capacity of a sufficiently large volume of superfluid helium very near to its critical temperature and to make accurate measurements of critical point exponents, in order to better test current theory.

b. The Zeno Experiment, which is designed to measure the large density fluctuations in xenon near the critical point that are responsible for the phenomenon of critical opalescence.

Gravity's role in the presence of very weak binding forces has not led yet to any readily identifiable experiments. However, the growth of protein crystals could be affected by convection phenomena, as in 1 a above, or by the more subtle action of gravitational forces that can distort macromolecules. Similarly, weak gravitational signals could influence the direction of the growth of plants (e.g., as in geotropism) or the development of cells.

Comments on experiments based on the influence of gravity in the presence of very large masses or for very long times, others such as Gravity Probe B, and other experiments similar to those mentioned in $4 \mathrm{a}$ and $4 \mathrm{~b}$ above are made in Space Science in the Twenty-First Century. ${ }^{1}$

Phenomena related to the role of gravity in structural members or over long distances are beyond the scope of this report, but they must be considered in the context of habitation and structures on the Moon or the planets. For example, the engineering of a building or a bridge on the Moon $(0.16$ $g_{E}$ ) might entail selecting materials that are entirely different from those that would be chosen for use on Earth.

\section{REFERENCE}

1. Space Science Board. 1988. Space Science in the Twenty-First Century: Imperatives for the Decades 1995 to 2015-Fundamental Physics and Chemistry. National Academy Press, Washington, D.C. 


\section{Status of the Field}

Microgravity research is a diverse multidisciplinary effort that has been subdivided into so-called disciplines. Each has been associated with a discipline working group (DWG) operated previously under the auspices of the Universities Space Research Association. Typically, each DWG has consisted of 5 to 10 interested scientists and engineers. All of the groups have been chaired by a person from outside NASA and cochaired by someone from a NASA center. These groups are biological sciences; combustion science; electronic materials; fluids, interfaces, and transport; fundamental processes; glasses and ceramics; and metals and alloys.

The CMGR has met with representatives of these DWGs, studied their reports, reviewed previous status and workshop accounts on the field of microgravity research, such as Materials Processing in Space, ${ }^{1}$ Microgravity Science and Applications: Report on a Workshop, ${ }^{2}$ and Review of Microgravity Science and Applications Flight Programs-January-March $1987,{ }^{3}$ and conducted independent investigations. This process has resulted in summaries of the status of each disciplinary area except fundamental processes, which, as has been indicated, is summarized in Space Science in the Twenty-First Century: Imperatives for the Decades 1995 to 2015Fundamental Physics and Chemistry; ${ }^{4}$ these summaries are presented in appendixes $A$ to $F$ of this report.

As regards the status of the field, the Committee on Microgravity Research reached the following conclusions: 
- The current subdivision of microgravity research into seven "disciplines," as represented by the DWGs, is obsolete and misleading. Thus the CMGR recommends that microgravity research be reorganized into the following six categories:

- Biological science and technology,

- Combustion,

- Fluid science,

- Fundamental phenomena,

- Materials, and

- Processing science and technology.

These six areas, as opposed to the former seven "disciplines," are chosen to reflect future opportunities more realistically. For example, combustion would entail consideration of both combustion science and fire safety; fluid science would address not only classical fluid dynamics, but also the mechanics of drop dynamics and the physics of free fluid boundaries; and processing science and technology would emphasize phenomena instead of a particular material and sometimes would be conducted on model materials of little practical value. Materials would encompass situations in which the material itself (whether metal, ceramic, glass, polymer, or composite), rather than a processing phenomenon, is the primary object of study.

- Development is more advanced in the DWG areas of fluids, interfaces, and transport; metals and alloys; and combustion science than in the other historical DWG areas.

- Studies in the area of biological sciences show promise in protein crystal growth, but little in other directions such as electrophoresis. However, current research activities in protein crystal growth are largely exploratory and empirical and hold out little hope of explaining why protein crystals grow differently in space or how to exploit such differences.

- Excellent, but only a few, experiments are being done in the area of fundamental phenomena. Future experiments are likely to materialize from unsolicited proposals rather than from NASA's Announcements of Opportunity (AOs).

- Research in the area of electronic materials has concentrated thus far on bulk materials and so is out of the mainstream of current technology, in which research on electronic properties focuses on the properties of thin films deposited on substrates. In some cases, however, the quality of the bulk crystals used as substrates can influence the properties of epitaxial films, and, in limited applications, bulk crystals are used as primary components. Therefore, some research on them is likely to be of practical value and could produce information of scientific importance to materials processing. 
- Glasses and ceramics constitute a relatively underdeveloped area at present; some research overlaps with that done in metals and alloys.

- Microgravity research is a relatively new and laboratory-intensive field that requires frequent access to space. To date, progress has been limited considerably by the paucity of flight opportunities.

\section{REFERENCES}

1. Committee on Scientific and Technological Aspects of Materials Processing in Space, Space Applications Board. 1978. Materials Processing in Space. National Academy of Sciences, Washington, D.C.

2. Solid State Sciences Committee, Board on Physics and Astronomy. 1986. Microgravity Science and Applications: Report on a Workshop. National Academy Press, Washington, D.C.

3. Review Committee, J. Robert Schrieffer, chairman. 1987. Review of Microgravity Science and Applications Flight Programs-January-March 1987. Universities Space Research Association, Washington, D.C.

4. Task Group on Fundamental Physics and Chemistry, Space Science Board. 1988. Space Science in the Twenty-First Century: Imperatives for the Decades 1995 to 2015-Fundamental Physics and Chemistry. National Academy Press, Washington, D.C. 


\section{The Conduct of Microgravity Research}

Some aspects of microgravity research are unique. Paramount is that much of it takes place in an environment remote from Earth and, therefore, will be practically inaccessible except when specific missions are flown. Coupled with this inaccessibility is the enormous cost of such research, both in initial investment and in operating costs, especially when humans are on board. Details of these distinctive aspects are addressed in the following sections.

\section{INSTRUMENTATION}

The conduct of microgravity research necessitates developing scientific equipment that is capable of withstanding the stresses of launch and reentry and of functioning reliably and safely in space. Although it is highly specialized, such equipment is distinguishable from other space hardware because of its functional diversity.

Generally, equipment serves one of two purposes: synthesis and processing of specimens or observation and measurement of phenomena. Important considerations include size and volume; means for the insertion, manipulation, removal, and storage of specimens; ambient atmosphere; thermodynamic (e.g., temperature, pressure, composition) range and sensitivity; observational capabilities (including photographic or video recording); power needs; thermal dissipation; and safety issues. This degree of complexity places exceptional burdens on design, operational reliability, accommoda- 
tion and integration in larger systems (e.g., the Shuttle or a space station), lead time needed for development and construction, and cost.

The interaction of users with this equipment is quite different from their interaction with many other space instruments. Whereas repetitive observations and large data sets are typical of space hardware in the observational sciences, the user of microgravity equipment must often interact with the equipment to change experimental parameters from run to run. Furthermore, the data from an experiment are often contained in a specimen that has been synthesized or processed, thus requiring complete characterization of the specimen before continuing with the next experiment. Sometimes statistical design of experiments involving multiple samples and varied parameters can be used to increase efficiency, but this requires reproducibility and parameters that are well controlled, a rarity for space experiments so far.

Finally, there is the question of multiuser versus specific-user equipment. In principle, general multiuser equipment would seem to have the advantage because it could be designed and built in advance and would be economical because of the large numbers of users served. In practice, this is not usually the case. Trying to design in advance equipment that will serve the anticipated needs of multiple users entails large amounts of engineering design and necessitates many compromises, often resulting in equipment that is satisfactory to no one and is extremely expensive. An alternative approach is to design and build equipment that is specific to a particular experiment or class of experiments, in close cooperation with the principal investigator(s). Although this approach requires long lead times and the resulting equipment many not be useful elsewhere, the equipment will satisfy the intended need; such an approach is thus a better overall strategy.

\section{MANNED VERSUS ROBOTIC INTERACTION}

It is possible to conduct microgravity experiments in a number of modes, the extremes being continuous manned interaction (similar to laboratory work on Earth) and robotic interaction (full intelligent automation). Each of these has advantages and disadvantages. Hybrid modes are also possible and usually more realistic. An optimum program will use a mix of modes that will depend on the set of experiments to be accomplished, the instantaneous state of technology, and cost-effectiveness.

Continuous manned interaction has the advantages of allowing for better observation and analysis of the experiment and better dexterity and flexibility in correcting any malfunctions, servicing, tuning, or adjusting for parameter changes. Unfortunately, this is the most expensive mode of operation, because it necessitates a life support system for humans and stringent 
safety standards for all of the equipment. Some human skills can be utilized by remote observation and control, with occasional access granted for major servicing and repair; of course, limited access can be a serious handicap for some experiments.

A free-flying spacecraft with a combination of robotic interaction and manned remote control can lead to a reduction in the stray vibrations or accelerations often identified as artificial gravity, or "gravity-jitter." Some of these result from robotic movements, comparable to those attributable to human movements. Disturbances from other experiments are possible; however, disturbances from docking, maintenance activities, and life support systems would be absent. Some experiments will not require extremely low levels of gravity, only well-monitored, reduced levels.

A fully automated (robotic) free-flying spacecraft can offer major cost advantages. Precise control of the cabin environment is not needed, and toxicity resulting from hazardous materials is a much less significant concern. Moreover, safety standards are considerably less stringent because catastrophes will not normally result in the loss of human life.

In summary, some experiments require the advantages of a space station laboratory but others can be done as well or better, and certainly at much less expense, in a free-flyer configuration.

\section{RANGE OF MICROGRAVITY FACILITIES}

Microgravity conditions can be found in a broad range of facilities, ranging from relatively simple ground-based drop tubes to complex facilities such as Spacelab on the Space Shuttle. The costs of these facilities vary from a few thousand to millions of dollars, and they differ in accessibility, ease of use, and utility. The experimenter's choice of these facilities should be based on specific research needs as well as return on investment. For certain experiments, a high-quality microgravity environment is essential. For others, a rather noisy, low-gravity environment will suffice. In some situations involving fluids, low gravity can be simulated by matching densities to reduce buoyancy forces, but this approach restricts greatly the set of fluid properties and the phenomena that can be studied. Since microgravity research is in an embryonic stage, it is difficult to rely on any one facility for experimentation.

Drop tubes and towers, aircraft flying parabolic trajectories, and sounding rockets provide test periods of several seconds to a few minutes. These facilities supply representative gravitational accelerations of $10^{-2} g_{\mathrm{E}}$ for the KC-135 airplane, $10^{-5}$ to $10^{-6} g_{\mathrm{E}}$ for sounding rockets, and $10^{-6} g_{\mathrm{E}}$ for drop tubes and are available to U.S. investigators at sites developed and operated by the government (see Figure 1). NASA's sounding rocket program, oriented toward astrophysics and space science payloads, has seen renewed 


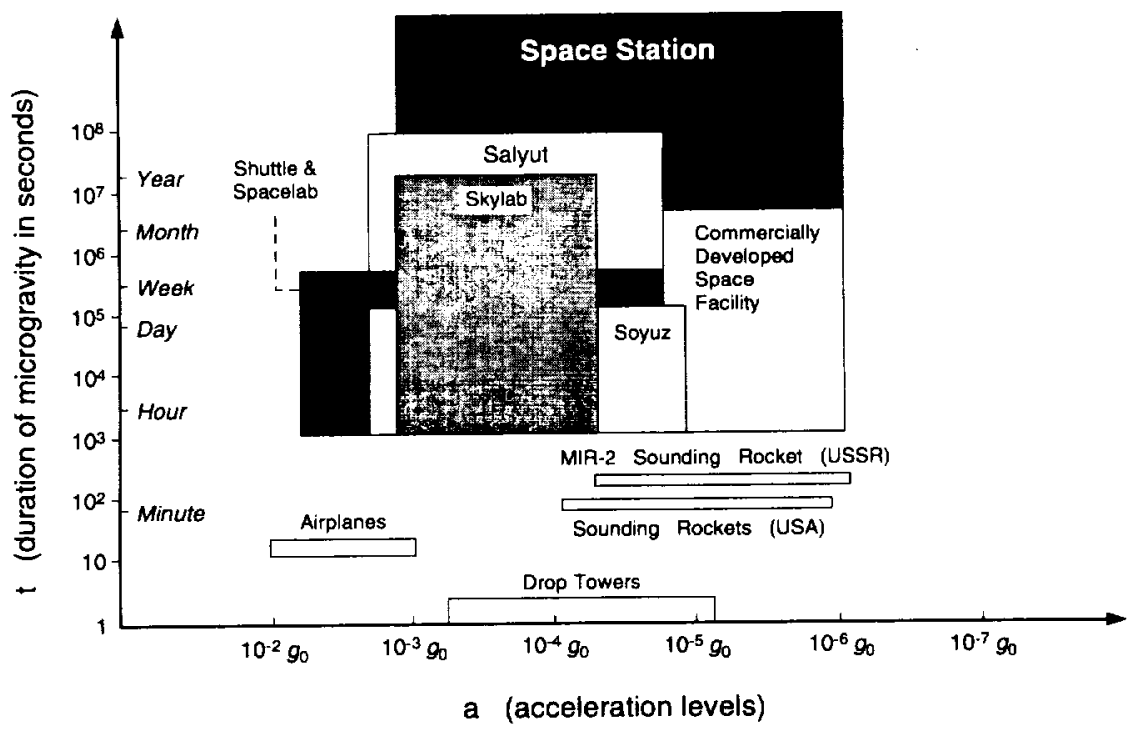

FIGURE 1 Characteristic duration and acceleration levels. SOuRCE: Microgravity Science and Applications Division, NASA.

microgravity usage under the direction of NASA's Office of Commercial Programs. A four-stage sounding rocket, with improved capabilities of up to 20 minutes of microgravity and a $1,500-\mathrm{km}$ altitude, is under consideration for members of the centers for the commercial development of space (these centers are discussed below, under "Commercial Programs"). Experiments in sounding rockets, managed by NASA's Office of Commercial Programs, were resumed in 1989.

Orbital facilities include the Space Shuttle, recoverable capsules launched on expendable vehicles, free-flying spacecraft, and space stations. These facilities offer enhanced resources in terms of volume, power, cooling, crew time, and data management, but, most importantly, they enable test times to be extended to days and perhaps weeks (for an Extended Duration Orbiter). Accelerations are in the $10^{-4}-g_{\mathrm{E}}$ range at the middeck of the Shuttle; this location has been used effectively by experimenters because of the ease with which the crew can be integrated and the potential ability to achieve late preflight and early postflight access. Dedicated pressurized Spacelabs, located in the payload bay of the Shuttle, can accommodate several experimenters working simultaneously. Missions having a large concentration of microgravity research experiments are scheduled to begin flying in 1992. Spacelab provides up to $7.7-\mathrm{kW}$ peak power for 15 minutes every 3 hours and $3.4-\mathrm{kW}$ maximum continuous power. Each flight can hold up to a $4,500-\mathrm{kg}$ payload, with an overall volume of $8 \mathrm{~m}^{3}$ available to users. These 
provisions greatly extend the researcher's ability to perform more experiments and to conduct experiments of longer duration with programmed distributions of power and crew time.

Choosing the most cost-effective, low-gravity experiment capability is very important. Experiments that can be flown on less expensive facilities should be flown on those facilities. For example, experiments that can be carried out adequately on sounding rockets should not be flown on Spacelab or Space Station Freedom.

The hundreds of hours of U.S. and other experiments conducted in orbit have offered limited insights into the myriad problems that exist. The limitations have been considerable in comparison to the research procedures performed regularly on Earth in other areas. Many failures have been caused by inadequate knowledge of the space environment; restrictions on the weight, size, power, experience, and expertise of the mission specialists and astronauts; and the quality and duration of the microgravity. Generally speaking, developments in microgravity research, as in any new field of research, will require additional thousands of test hours in order to achieve reproducibility, process control, improvements, and verification of results.

Given the high costs, long lead times, and uncertainties involved in developing and sustaining both the facilities and the scientific interest of this research community, it is clear that government funding for access is indispensable, as it is for development of the instruments and for rigorous selection of the experiments. The committee recommends that a concerted effort be made to classify experiments according to their minimum needs in order that the most cost-effective access to reduced gravity will be used.

\section{MICROGRAVITY RESEARCH OUTSIDE THE UNITED STATES}

Programs in microgravity research are conducted by foreign countries also, most notably the member nations of the European Space Agency (ESA), the former USSR, and Japan. Europe (the ESA and particularly Germany) holds a strong position in microgravity research compared with the United States. After a greater initial investment by the ESA and Germany, Europe continues to fund microgravity research at a level equivalent to U.S. funding. The Europeans have demonstrated greater efficiency in obtaining both quantitative and qualitative results to date and hold a substantial lead in the design of microgravity experimentation instrumentation, for example, Mephisto (a solidification furnace) and space bioseparation (for biological purification). Europe's dependence on the United States for access to space has been a weak point in its microgravity research. Because of the lack of NASA flight opportunities, the Europeans have established an extensive 
program of short-duration experiments with a diverse infrastructure (i.e., drop towers, aircraft for parabolic flights, sounding rockets, and high-altitude balloons).

The former USSR put the MIR space station into orbit in 1986 and has had opportunities to conduct microgravity research for long durations (on the order of years) and with extensive manned interactions. From sketchy reports of the results and discussions with a few former Soviet researchers, this committee has concluded that this program, with minor exceptions, mirrors that of the West.

The Japanese government and industry have targeted reentry capsules as a strategic technology for development and acquisition during the 1990s. Japan's National Space Development Agency and its Ministry of International Trade and Industry are monitoring the U.S., German, and ESA programs closely with the intention of entering into collaborative arrangements, for example, development of the Japanese Experiment Module for Space Station Freedom. The Japanese also have their own programs involving sounding rockets and parabolic aircraft flights.

\section{COMMERCIAL PROGRAMS}

Commercial engagement in microgravity research began in 1980 with the initiation of three new legal vehicles for joint government-industry association on a no-exchange-of-funds basis. The general concept is that industry is to supply the experimental capabilities while NASA is to provide the spaceflight opportunities. The goal is to encourage the early involvement of industry in microgravity space experiments that would lead to its long-term investment in using the space environment for commercial purposes. A list of the joint arrangements as of 1989 can be found in the NRC's Report of the Committee on a Commercially Developed Space Facility. ${ }^{1}$ As of the present time, no commercial endeavors have matured to the stage that there are full paying customers for space transportation and operation services.

In addition to space experiment incentives, NASA's Office of Commercial Programs, in cooperation with industry, has established centers at several universities for the development of commercial space experiments. ${ }^{2}$ Started in 1986, these centers for the commercial development of space (CCDS) were given a five-year period in which to become independent by attracting increased industrial funding.

The NRC Committee on a Commercially Developed Space Facility proposed a number of space facilities that would enable further initiatives in commercial microgravity experiments. ${ }^{3}$ In the late 1980 s, some proposals were made by industry to supply a free-flying facility that would accommodate microgravity experiments in return for a guarantee by NASA to lease 
the facilities for government use as well. However, the NRC committee commented that ". . . having greatly enhanced access to space up to five years earlier than the Space Station is anticipated actually would add little toward speeding space commercialization based on exploitation of the microgravity environment" (p. 55). Hence, the commercially developed space facility concept was not pursued by NASA.

Overall, the various NRC committees reviewing the microgravity program have not envisioned any near-term commercial opportunities. ${ }^{4,5}$ No indications surfaced in 1991 to change these views based on the results of NASA's program or the foreign programs.

Some disturbing trends are becoming evident in the commercial programs for microgravity experiments. There is competition between scarce spaceflight opportunities and the university research funded by NASA's Division of Microgravity Science and Applications. The zeal to provide commercial "customers" priority access to space may jeopardize opportunities for worthy space experiments that have been on hold for a long time. Furthermore, although commercial experiments are regarded by the centers and their sponsors as proprietary and therefore not subject to peer review, there is an increasing suspicion that their quality is lower than that of traditional university research. This suspicion arises because some companies have not pursued their investment in the joint agreements and have not published their results, even though they have no further commercial interest in the work. Work that has appeared has not been published in the archival, peer-reviewed literature.

Accordingly, the CMGR recommends that a thorough technical review of the centers for the commercial development of space be conducted to determine the quality of their activities and to ascertain to what degree their original mission has been accomplished.

Another committee has concluded that the microgravity environment should "be considered primarily as a tool for research and secondarily as a manufacturing site" since "significant demands for manufacturing opportunities are unlikely in the near term" (p. 1). ${ }^{6}$

The CMGR agrees with this assessment and believes that a strong and comprehensive base of research and technology in the microgravity environment must be developed first, on the same basis that it is developed on the ground, before any real commercial opportunities can arise. Furthermore, these opportunities will be primarily in the use of space to understand and improve ground-based manufacturing processes that are sensitive to fluid disturbances of the type that can be studied in space.

Finally, the CMGR notes that materials processing and manufacturing in space for the purpose of sustaining space travel and human exploration 
probably will become more important eventually than materials processing and manufacturing in space for providing products for use on Earth.

\section{THE RESEARCH AND ANALYSIS PROGRAM}

The research and analysis (R\&A) program in NASA's Microgravity Science and Applications Division consists of the ground-based research needed to provide the context of knowledge from which the flight program originates as well as the infrastructure required to analyze microgravity experiments in a broader context.

The microgravity science program has been rooted historically in the materials processing sciences, with the inclusion of some additional components such as fluid flow, combustion, critical-point phenomena, and biotechnology. Many research activities have focused on the modeling and understanding of very subtle phenomena in the fluid state. This work has contributed profoundly to our understanding of fluid behavior and its effects on materials preparation on Earth. However, NASA's R\&A program of the future must capture a broader range of interests in physics and chemistry.

In addition to expanding the base of the R\&A programs, the microgravity program must improve its effectiveness and productivity. The current program includes investigators who have been funded for 13 years in some cases. In addition, many of these investigators have experience with only one or two space experiments. Therefore, the overall health of microgravity science is arguably poorer than that of other comparable scientific endeavors with faster development cycles. The current research program should be reconstituted and refocused in order to revitalize it and to open up new opportunities.

The CMGR recommends that NASA apply a set of value criteria and measurement indicators to define the research and analysis program more clearly.

Some of the value criteria and indicators that should be used for the new R\&A program are as follows:

- Value criteria

- relevance to other science

- significance of potential contributions to important scientific questions

- past experience and track record of investigators

- ability to develop adequate plans and well-understood requirements for space experiments

- scope of effort needed to achieve significant results. 
- Indicators

- innovation - novelty, uniqueness

- quality - for example, publication in refereed technical and scientific literature

- productivity - for example, the length of time taken to execute the experiments in space as compared to a ground-based equivalent; also, the percentage of time that space hardware is actually used

- cost-effectiveness-including consideration of hardware development costs, reusability, reconfigurability of hardware, and integration costs on different space vehicles.

These value criteria and indicators should be evaluated in the context of other areas of physical and chemical sciences to determine the relationship between funding levels and research output over a reasonable period of time (such as three years).

The CMGR recommends that the funding level for research and analysis in microgravity science be established as a fixed percentage of the total program of NASA's Microgravity Science and Applications Division in order to build a strong scientific base for future experiments.

Past experience indicates that this percentage should be between 30 percent and 50 percent for new science. This percentage dropped from 50 percent in 1981 to 13 percent in 1991 as space hardware expenses grew. There are plans to double the R\&A budget over the next five years, which is a step in the right direction; however, the size of the total program is increasing at least at this rate or faster, so that the percentage increase of $R \& A$ is hardly changing. If more research of higher quality and wider diversity is to be incorporated into the microgravity program, it is imperative that the $R \& A$ budget be a larger fraction of the total microgravity budget of NASA's Microgravity Science and Applications Division.

\section{REFERENCES}

1. Aeronautics and Space Engineering Board. 1989. Report of the Committee on a Commercially Developed Space Facility. National Academy Press, Washington, D.C.

2. Space Applications Board. 1988. Industrial Applications of the Microgravity Environment. National Academy Press, Washington, D.C.

3. Aeronautics and Space Engineering Board. 1989. Report of the Committee on a Commercially Developed Space Facility. National Academy Press, Washington, D.C. 
4. Aeronautics and Space Engineering Board. 1989. Report of the Committee on a Commercially Developed Space Facility. National Academy Press, Washington, D.C.

5. Committee on Scientific and Technological Aspects of Materials Processing in Space, Space Applications Board. 1978. Materials Processing in Space. National Academy of Sciences, Washington, D.C.

6. Aeronautics and Space Engineering Board. 1989. Report of the Committee on a Commercially Developed Space Facility. National Academy Press, Washington, D.C. 


\section{Toward the Development of a Research Strategy}

The Committee on Microgravity Research recommends that a longterm research strategy, such as that developed by each of the Space Studies Board's other discipline committees, be developed for microgravity science. The strategy should be based on the following considerations:

1. It should be recognized that, to date, no examples have been found of materials that are worthy of manufacture in space. Unless and until such examples are found, space manufacturing of products to be used on Earth should be deemphasized as a reason for undertaking microgravity research.

The descriptor "materials processing" should not be used for NASA's budget line item because it is misleading. The CMGR recommends that "microgravity research" be used instead.

2. The main rationale for the microgravity research program should be to improve our fundamental scientific and technological knowledge base, particularly in areas that are likely to lead to improvements in processing and manufacturing on Earth. A secondary rationale should be to develop the technologies for handling materials in space and possibly for processing materials to be used in space.

3. The $g$-level (acceleration vector environment) must be measured accurately, locally, frequently, and synchronously with every experiment. These data must be provided to each principal investigator immediately.

4. Experiments that appear to have produced promising results should 
be repeated to examine reproducibility. Most results to date are the product of single experiments.

5. A number of examples illustrate clearly that microgravity makes a measurable difference. The question is: Why? Critical experiments should be designed and conducted to learn why certain phenomena occur in microgravity, not merely to exhibit that they do occur.

6. A thorough program of ground-based research must precede and succeed every microgravity flight. Much of this research will form a context for the flight program but will not be directly related to a flight experiment. Such a context is very important because of the high relative cost of flight experiments. Points 7 to 9 below pertain to this ground-based program.

7. Much more effort needs to be made to model phenomena suggested by microgravity observations. In many cases, models are obsolete (e.g., for eutectic growth) or nonexistent (e.g., for protein crystal growth).

8. The materials used in microgravity experiments should be characterized thoroughly both before and after flight. The thermophysical data needed to interpret experiments should be measured as a part of the program if they are unavailable from the literature.

9. Whenever exemplary materials are produced in microgravity, considerable effort should be made to replicate them through Earth-based research, which is much less expensive and might lead to commercial applications.

10. The interest in electronic materials today is in thin films; bulk electronic materials are of secondary importance and should be studied in microgravity only if their quality as substrates or primary components is important or to the extent that such studies can yield fundamental knowledge about processing.

11. Research opportunities should involve a planned budgeted balance of focused opportunities, some communicated by an Announcement of Opportunity (AO) from NASA and others funded on the basis of unsolicited proposals submitted to NASA. The field is still very young, and so many good ideas are yet to come. If AOs are used to distribute too much of the funding, growth of the best ideas will be stifled, and the field will not be intellectually exciting to the scientific community.

12. The funding level for research and analysis in microgravity science should be established as a fixed percentage of the total program of NASA's Microgravity Science and Applications Division in order to build a strong scientific base for future experiments.

13. NASA and its advisory committees should analyze and classify experiments according to their minimum facility requirements so that they can be carried out in the most cost-effective manner. From this classification, candidate experiments for drop towers, free-falling aircraft, sounding rock- 
ets, the Space Shuttle, space stations, or free-flying spacecraft can be identified.

14. Most microgravity equipment should accomplish specific experiments and should be designed and built in close cooperation with the principal investigator(s). The designing and building of multiuser facilities for anticipated experiments should be avoided.

15. The centers for the commercial development of space should be reviewed technically to ensure their quality and to ascertain to what degree their original mission of becoming independent through industrial funding has been accomplished.

Based on the above points, a strategy for microgravity research should include:

1. A definition of the overall goals of the microgravity science field;

2. A summary of the current knowledge that is related to strategic considerations;

3. A statement of the fundamental questions that need to be answered;

4. The overall strategy and its rationale, derived from and based on the fundamental questions and the ability to address them;

5. The primary scientific objectives ranked in order of their priorities (together with the value criteria used);

6. A set of experiments required to achieve the stated objectives;

7. Definition of the requirements for such experiments and their accommodation in various facilities and low-gravity experiment modes, which reduces total cost and enhances the productivity of experiments;

8. Other resources needed for a successful scientific program, such as access to space, operational support, and ground-based research;

9. The indicators for a successful scientific program, for example, comparison with accomplishments attained during ground-based equivalents of experiment time, equipment utilization, and so on. 
APPENDIXES 



\section{Biological Sciences}

Biotechnology encompasses the sciences based on molecular biology and the engineering and technological developments needed to convert discoveries into useful products. The biotechnology field is one of the most rapidly developing and widely ranging areas of research. Typically, the biological research considered for microgravity is divided into the study of isolated biomacromolecules (proteins and nucleic acids) and their assemblies, organelles, and cells in controlled fluid and chemical environments. According to the NASA DWG on biological sciences, the microgravity environment offers advantages for (1) examining the physical chemistry of biomolecular structures and their interaction, most specifically by protein crystallography; (2) using separation processes to provide improved sensitivity in preparative and analytical techniques; and (3) studying cells and cell cultures.

To date, there has been only slight progress in the application of microgravity sciences to any of these thrusts in the biological sciences. Hence, most of the discussion that follows is based on prospects and not on completed research.

\section{STATUS}

\section{Protein Crystallography}

Protein crystallography is the general name given to determination of the detailed, three-dimensional structure of biological macromolecules, in- 
cluding proteins, DNA, and RNA, by using $x$-ray crystallographic techniques. The determination of accurate macromolecular structures is absolutely necessary for establishing the molecular mechanisms of biological reactions, for rational drug design (in which a molecule is designed to bind to a specific target protein), and for the design of proteins and nucleic acids with new activities and functions. During the past decade, the methods of protein crystallography have been made faster and more accurate through the use of improved data collection methodologies and more powerful computers. Now, virtually all proteins and nucleic acids can be made in sufficiently large quantities for crystallographic analysis using the methodologies of cloning genes or by direct chemical synthesis. This means that any biological macromolecule for which there is a gene sequence can, in principle, become a subject for study by protein crystallography. This will become increasingly important as large numbers of sequences for proteins of unknown structure and function become available through the efforts of the Human Genome Project. The amino acid sequence of a protein of either known or unknown function cannot be interpreted usefully in the absence of a three-dimensional structure.

Determination of crystal structure by protein crystallography requires well-ordered, single crystals whose minimum dimension is 0.2 to $0.4 \mathrm{~mm}$ and whose maximum dimension need not be larger than $1 \mathrm{~mm}$. An optimal crystal size for most biological macromolecules would be about $1 \mathrm{~mm}$ in all three dimensions. The intensity of the diffraction pattern increases as the cube of the linear dimension increases up to this optimal size. Thus, crystals much smaller than $0.1 \mathrm{~mm}$ in maximum dimension are not useful for crystal structure determination because the $x$-ray diffraction pattern that results from such a crystal is too weak to be measured accurately.

The accuracy of the resulting coordinates derived from determining a crystal structure is directly related to the resolution to which the crystals diffract. The resolution is quoted in terms of the minimum spacing in angstroms between Bragg planes that can be resolved. The ratio of measurable data to refinable parameter increases as the inverse cube of the resolution of the measurable diffraction pattern; that is, there are eight times more data at $2-\AA \AA$ resolution than at $4-\AA$ resolution. Proteins whose structures are refined at $2 \AA$ have coordinate errors of 0.2 to $0.3 \AA$ in favorable cases. Structures determined at higher resolutions have smaller errors in atomic coordinates, while those determined at lower resolutions can have significantly larger errors.

During the past 10 to 15 years, new techniques of crystallization, particularly microcrystallization, have greatly improved the speed, yield, and quality of crystals grown in various laboratories around the world. In turn, these improvements in crystallization methodologies have increased immensely the usefulness and impact of protein crystallography in the biological com- 
munity in general. It is very probable that any general improvement in the ability to grow suitable crystals of macromolecules will have a major impact on crystallography's usefulness to the biomedical community.

\section{Separation Processes}

In many instances, progress in the biological sciences and in biotechnology is limited by the ability to separate a myriad of proteins, nucleic acids, and associated complexes created by modern genetic engineering either as natural mixtures in blood serum or from manufactured mixtures, such as cell culture media, hybridoma cultures, or synthetic solutions. Many of the separation processes currently in use are affected deleteriously by gravity. For example, buoyancy-driven convection disturbs sedimentation processes.

To counterbalance the action of gravity, the most common method used for analytical separations is electrophoresis carried out in water-based gels. The gel serves to limit hydrodynamic convection because it causes muchreduced separation rates.

A major microgravity research program has focused on the study of liquid-based electrohydrodynamics, which has been shown to limit the scaleup in space of electrophoresis experiments for a given separation efficiency.

Other bioseparation methods also have received attention, for example, isoelectric focusing used in protein analysis. Again, the electrohydrodynamic effects have been found to be important. Other methods include phase separation based on chemical partitioning. The efficiency of these methods hinges on the ability to maintain a very dispersed phase and is influenced strongly by sedimentation.

\section{Cells and Cell Cultures}

Current biological research includes the formation of ordered biocompatible materials, genetic manipulation, cell fusion, and the regulation of cell growth and differentiation. One of the most challenging aspects of these problems is the manipulation of fragile mammalian and plant cells, which are very sensitive to hydrodynamic shear forces and to interactions with container walls, and have complex metabolic requirements.

The microgravity environment has potential for the conduct of cell science research. Ground-based research is under way to characterize the fluid dynamic environment of cell culture systems and includes studies of the hydrodynamics of cell suspensions during separation. The influence of hydrodynamic, cell-cell, and cell-container interactions on setting upper limits of cell densities needs to be determined. New devices for cell cultures are designed to provide low shear rates and minimal sedimentation. To date, the viability of these concepts is unproven. 
Finally, the fusion of cells to produce viable hybrid cells is another technology that may benefit from microgravity. Cell fusion requires the interaction of two cell types and the annealing of the cell membrane to yield a single cell; usually, this is a low-frequency event in current experiments on Earth. The cells generally have different densities, and so sedimentation tends to separate the cells during fusion. It has been hypothesized that a microgravity environment would alleviate these tendencies and lead to higher fusion rates. Support for this hypothesis has come from experiments on the German Spacelab and on TEXUS sounding rocket flights; both exhibited fusion in microgravity of yeast cells.

\section{MAJOR RESEARCH ACCOMPLISHMENTS}

Use of the microgravity environment is just beginning to increase our understanding of the biological sciences and to enable us to develop innovative biotechnological processes that can exploit microgravity. Several research projects have resulted in valuable findings in microgravity crystallization and protein separations.

Thus far, the NASA-sponsored microgravity crystallization efforts have had two major components. One is a ground-based, systematic examination of the principles and methodologies of protein crystallization. Although these studies are still in their infancy, they show considerable promise and constitute one of a relatively small number of significant systematic approaches to protein crystallization being carried out in the world.

The second major effort has been in the growth of protein crystals in space. In addition to NASA's efforts in space, experiments are being carried out by the Europeans and the former Soviets. While the number of experimental examples has been limited, the general conclusion drawn from these crystallization experiments in space is that it is possible to grow larger and morphologically better protein crystals that diffract to higher resolution, in at least some cases. In a recent NASA experiment, crystals of three proteins grew large enough to be examined by $x$-ray diffraction, and, in each case, the crystals diffracted to higher resolution than the best Earthgrown crystals. In these examples, the amount of measurable data seems to have increased by about a factor of two. In about 40 percent of the crystallization experiments, no crystals at all were obtained, and in another 30 percent, the crystals were too small for $x$-ray analysis. This is attributed, probably correctly, to the crystallization conditions being suboptimal in the specific microgravity environment used and to the small number of separate crystallization experiments done in the case of each protein.

In another program, the potential for achieving high precision in electrophoretic separations has been addressed in microgravity experiments. Separations carried out on Earth and in space, coupled with extensive theo- 
retical analysis, have shown the important role of electrohydrodynamic forces in determining the efficiency of separation; the convective movement of charged molecules in an electric field leads to significant convective mixing in large-gap electrophoresis devices in space and severely degrades the quality of the separation. These effects are masked on Earth by the smallgap devices that must be used to prevent convection due to density gradients. This research exemplifies the role of microgravity experiments in determining the ultimate limits of various separation technologies for veryhigh-value-added materials.

\section{RESEARCH PROSPECTS AND OPPORTUNITIES}

Imaginative research should be supported in both science and engineering applications. Research should be sponsored in cell science, bioseparation, and protein crystallization. The last topic has perhaps the most potential for an immediate impact on ground-based research.

Protein crystal growth is one of the most promising possibilities. An important overall goal of both ground-based and microgravity efforts should be to find factors that exist in crystallization under microgravity conditions in space that are important in yielding the larger, better-formed crystals that diffract to higher resolution and to try to the extent possible to duplicate those conditions on Earth. This would require additional studies of the crystallization process in ground-based laboratories. While the present work on mechanisms of protein crystallization being conducted at the University of Alabama's Center for the Commercial Development of Space is well regarded, an incremental expansion of such fundamental studies would be done most effectively through competitive research grants that could be made available to any of the crystallographic laboratories in the United States. Obtaining the active participation of a larger number of protein crystallographic laboratories in this country on this fundamental aspect of the field of protein crystallography would be most likely to yield important results.

The present plans for experiments that are to be performed in space to study the crystallization process and to vary the conditions of crystallization should be continued and expanded. Only by monitoring the crystallization process in space will it be possible to assess the ways in which microgravity and its effects on transport favorably affect the crystallization process. If this could be coupled with the ability to vary crystallization conditions while in space, it might be possible to increase greatly the yield of crystallographically suitable crystals obtained in space.

If the reliability of obtaining large, crystallographically suitable crystals in space could be increased considerably by on-board assessment, the method could become more generally feasible and useful to ongoing crys- 
tallographic studies. In general, only a small number of large crystals is required to obtain a high-resolution diffraction data set. Most of the crystals required to establish the protein structure could initially be Earth-grown crystals. The use of space-grown crystals to improve the accuracy of structures found using Earth-grown crystals could prove to be a more costeffective way to use this limited resource. It has not been demonstrated that the growth of crystals in space is the fastest or most cost-effective way of obtaining large crystals from small ones, although the on-board examination of crystal growth could change that conclusion.

Up to the present, the rationale for putting $x$-ray data collection equipment into space is less convincing. Building the capacity to analyze crystals in space would be extremely expensive, and it is not at all obvious that the general benefit would be commensurate with the very high cost. The reasons for evaluating crystals in space include the speed with which the results of crystallization could be evaluated, the ability to analyze a small number of crystals that might not be stable for the period of time required to return them to Earth, and the small possibility that some crystals might have their crystallinity distorted by gravity.

The promise of protein crystallography and the potential usefulness of microgravity in producing protein crystals of superior quality should not provide any part of the justification for building a space station. Growing crystals of superior quality in space is not close, nor is it likely to become close, to being cost-effective. All proteins and nucleic acids are highly polymorphic in their ability to form crystals. In other words, these biological macromolecules can be crystallized in many different crystal forms under different crystallization conditions; the presence or absence of gravity is merely one of many variables that can be explored in the pursuit of better crystals. Different crystal forms of the same protein frequently differ dramatically in the resolution to which they diffract $x$ rays-from $20-\AA$ to $2-\AA$ resolution. It currently is (and is likely to remain) faster and very much less expensive to obtain superior-quality crystals by changing the form of a crystal as a result of varying crystallization conditions on the ground, rather than by improving the form of an existing crystal through growing it in space.

While improvements in separation processes continue to be important to the study of biological systems, progress in ground-based separations has proceeded at a great pace and probably will have an overwhelmingly greater impact on biomedical research than separation methods in space will have. 


\section{Combustion Science}

\section{STATUS}

Combustion is a vital subject. For the foreseeable future, well over 90 percent of the U.S. energy supply will come from energy conversions that involve the high-temperature oxidation of fossil fuels. The world economy is affected by the efficiency of combustion processes in converting chemical energy to work or heat; the health of people and a balanced ecology depend on the reduction of pollutants emitted during combustion; and the viability of many transportation systems requires that combustion engines have a favorable power-to-weight (or thrust-to-weight) ratio.

The general subdiscipline of combustion science is very active. Three journals deal solely with combustion topics. Other journals in the fields of fluid mechanics, heat and mass transfer, chemical physics, thermophysics, propulsion and power, spectroscopy, applied mathematics, and environmental science regularly contain articles relating to combustion. Several international research conferences are dedicated to combustion topics. The Combustion Institute, the leading international research organization in the field, holds the premier international conference and supports scores of national and regional meetings. Combustion is the topic of many sessions at major annual conferences of the American Institute of Aeronautics and Astronautics, the American Society of Mechanical Engineers, and the Society of Automotive Engineers. Other societies address combustion topics occasionally.

Microgravity experimentation in combustion offers a variety of simplifications compared to experiments done at Earth's gravity. They include a 
reduction of dimensionality (the tendency toward spherical symmetry), the inhibition of settling effects for multiphase phenomena, and a substantial reduction of buoyancy forces. These simplifications provide the impetus for microgravity combustion research. Some challenges arise in this research, such as the emergence of surface tension as a major driving mechanism in certain two-phase experiments and the difficulty of achieving compact arrangements for forefront optical diagnostics. These challenges appear surmountable if given appropriate attention.

Several experimental programs are under way to study combustion in microgravity. For the most part, these programs have been successful, and some interesting and useful results have been obtained. Until recently, the microgravity program has not been visible to a wide community of combustion researchers. However, recent efforts by the Combustion Science Disciplinary Working Group have attracted an unusually large number of interesting research proposals to NASA's headquarters, and more research funds are now being committed in this area.

Opportunities in microgravity science have not been utilized at more than a fraction of their potential by the combustion research community. Gravity affects many combustion processes, especially in a laboratory simulation in which certain length scales are increased in order to improve resolution. Control of the acceleration environment offers the prospect of scientific breakthroughs in several areas of combustion science.

\section{MAJOR RESEARCH ACCOMPLISHMENTS}

Generally, the acceleration environment is found to have not only observable but also profound effects on material flammability characteristics, flame spread rates, and burning rates. This environment offers the possibility of addressing gravity-dependent variations to gain insights about fundamental processes. There is also the obvious research needed to develop new fire safety standards for spacecraft; the practice of applying in space standards developed and tested at Earth's gravity should not be continued.

Flame spread rates over solid fuels have been found to decrease as the ambient oxygen concentration decreases. ${ }^{1,2}$ Interestingly, dependence on the magnitude of gravity increases as the concentration of oxygen decreases. A flammability limit for oxygen concentration has been found, below which flames cannot propagate or exist. Furthermore, the magnitude of this oxygen concentration limit does depend on the level of gravity. The existence of this lower limit is a fascinating scientific finding that has a potential for extraordinary technological impact on fire safety issues, including spacecraft fire safety.

Forced air velocities have also been shown to affect spread rates. At low spread rates, radiative losses dominate the effects of transport rates that 
influence spread rates. Therefore, quenching occurs. Flame spread rates increase with increasing velocity to a point. Beyond that point, high velocity causes a blow-off of the flame.

In the case of ignition and flame spread in a gaseous combustible mixture above a pool of liquid fuel, the effects of buoyancy and of surface tension as a driving mechanism have been shown to be important. ${ }^{3}$

Flammability limits for gaseous combustible mixtures also have been found to depend on the magnitude and orientation of gravity. ${ }^{4,5}$ Natural convection tends to "stretch" flame fronts; that is, flame curvature and flow divergence are affected by natural convection. This stretch modifies the rate of transport across the front.

It has been demonstrated that flame propagation through particle clouds is sensitive to the motion of the particles. ${ }^{6}$ Microgravity environments are useful in this case to avoid settling and to maintain strict control of the density of the particles.

The buoyancy effect is predicted to have a stabilizing influence on flames. At reduced gravity, flames become unstable and cellular structures are formed, according to the theory. Cellular structures have been found experimentally at reduced gravity levels for lean hydrogen-air mixtures.

Experiments indicate that jet diffusion flames become larger, more spherical, and sootier as gravity is reduced, but the mechanism is not yet fully understood. $^{7}$

Preliminary data on spherically symmetric fuel droplet vaporization and burning support the theoretical prediction of the importance of certain unsteady effects. ${ }^{8}$ Buoyancy tends to destroy spherical symmetry, making the microgravity environment particularly appealing. Experiments in drop towers have indicated a new aspect of formation of a soot layer between the droplet and the oxidation flame. It has been suggested that thermophoresis is important in stabilizing this layer in the Stefan flow.

\section{RESEARCH PROSPECTS AND OPPORTUNITIES}

There are many opportunities for using microgravity facilities to do frontier research in combustion. Turbulent combustion, for example, is the area most in need of further fundamental characterization. The mixing that precedes a chemical reaction and that often is rate controlling occurs on very-small-length scales in practical situations. The resolution needed is not attainable in those cases. Theoretically, similitude can be maintained by decreasing velocities and increasing length and time scales within the proper relationship. However, at the larger-length scales, buoyancy can become important, destroying the similitude. Therefore, the microgravity environment offers an exciting opportunity for the study of turbulent-reacting flows with large scales, yet negligible gravity effects. 
Elimination of settling effects enhances conditions for the study of soot formation and agglomeration. The absence of settling also allows for useful studies of spray combustion and particle cloud combustion, as well as investigation of propagation rates and flammability limits. Extinction limits and details of the transient characteristics for an individual burning droplet and for an array of droplets burning together are also topics of interest.

More experiments on the spread of flames adjacent to liquid or solid fuels are required. In particular, it is necessary to understand the individual influences of buoyant, forced, and Stefan flows on spreading rates and on detailed flow field characteristics. Experiments with separate controls for each of the three influential types of flow are desirable.

A series of experiments on flame propagation through gaseous combustible mixtures is needed in order to evaluate the relative effects of various mechanisms on flammability limits. The consequences of chemical kinetics, radiative heat losses, fluid dynamical strain, Lewis number variation, and buoyancy should be determined. The consequences of these same parameters on flame front instabilities and cellular flame formation are also of interest. Access to a microgravity environment to separate the buoyancy effect from other potentially important effects is obviously useful.

The stabilization mechanism for a jet diffusion flame calls for further study. The roles of buoyant flows and diffusion should be determined, especially near the burner rim where the flame-holding action occurs.

The Combustion Science DWG has given the highest scientific priority to turbulent-reacting flows and the next highest to heterogeneous combustion. ${ }^{9}$ Laminar homogeneous combustion receives the third priority. With regard to applications, spacecraft fire safety receives the highest priority.

Combustion experiments will require a range of sophisticated instruments; more study is needed to obtain appropriately compact optical diagnostic equipment. Further examination of the allowable levels of artificial gravity is required.

\section{REFERENCES}

1. Olson, S., P. Ferkul, and J. T'ien. 1989. "Near-Limit Flame Spread Over a Thin Solid Fuel in Microgravity." Pp. 1213-1222 in Proceedings of the Twenty-Second Symposium (International) on Combustion. Combustion Institute, Pittsburgh.

2. Bhattacharjee, S., R.A. Altenkrich, S.L. Olson, and R.G. Sotos. 1988. "Heat Transfer to a Thin Solid Combustible in Flame Spreading at Microgravity." Proceedings of the 1988 Fall Technical Meeting on Chemical and Physical Processes in Combustion. Eastern Section, Combustion Institute, Clearwater Beach, Fla., December 1988.

3. Abramzon, B., D.K. Edwards, and W.A. Sirignano. 1987. "Transit, Stratified, Enclosed Gas and Liquid Behavior with Concentrated Heating from Above." Journal of Thermophysics and Heat Transfer 1:355-364.

4. Ronney, P.D. and H.Y. Wachman. 1987. "Effect of Gravity on Laminar Premixed Gas Combustion I: Flammability Limits and Burning Velocities." Combustion and Flame 62:107. 119. 
5. Strehlow, R.A., K.A. Noe, and B.L. Wherley. 1987. "The Effect of Gravity on Premixed Flame Propagation and Extinction in a Vertical Standard Flammability Tube." Pp. 1899-1908 in Proceedings of the Twenty-First Symposium (International) on Combustion. Combustion Institute, Pittsburgh.

6. Berlad, A.L., H.D. Ross, L. Facca, and V. Tangirala. 1990. "Particle Cloud Flames in Acoustic Fields." Combustion and Flame 82:448-450.

7. Edelman, R.B. and M.Y. Bahadori. 1987. "Effects of Buoyancy on Gas Jet Diffusion Flames, Experiment and Theory." Acta Astronautica 13:681-688.

8. Shaw, B.D., F.L. Dryer, F.A. Williams, and J.B. Haggard. 1988. "Sooting and Disruption in Spherical Symmetrical Combustion of Decane Droplets in Air." Acta Astronautica $17: 1195$.

9. Office of Space Science and Applications, Microgravity Science and Applications Division. 1990. Microgravity Program Strategic Plan 1990. National Aeronautics and Space Administration, April. 


\section{$\mathrm{C}$ \\ Electronic Materials}

\section{STATUS}

The field of electronic materials encompasses the understanding and control of microstructure and composition to achieve the electrical and optical properties required by high-performance computer and communications systems. Most work today is performed in silicon- and gallium arsenidebased systems in which device microstructures must be fabricated over progressively smaller dimensions. Thus the major research questions center on the measurement and manipulation of materials to realize device and circuit microstructures down to the level of atomic monolayers. Another emerging area is the use of micromachining methods to fabricate semiconductors for such applications as sensors and detectors. In these areas, materials preparation and synthesis techniques revolve mainly around thin-film deposition and patterning. None of these techniques benefits from the study of phenomena under microgravity conditions because of the inherently small dimensions, reduced pressures, and relative insensitivity to gravitational acceleration disturbances.

However, the preparation of bulk semiconductor and optical crystals has an important second-order influence on the control of microelectronic materials properties. Bulk crystals serve as substrates for epitaxial-film deposition and/or growth and in limited applications for the direct processing of devices and integrated circuits. The major research questions here include contamination and gettering effects, compositional uniformity, wafer surface flatness and/or defects, and electrically active, structural micro- 
defects. In the industrial growth of bulk crystals, compositional control is mostly achieved through control over segregation and convection phenomena at the growth interface. For these phenomena, gravitational acceleration exerts a major influence through interaction with fluid density gradients caused by inhomogeneities of temperature or composition. Moreover, studies in microgravity have the potential to elucidate the nature of these phenomena at $g_{E}$, so that they can be controlled more effectively on Earth.

Another important influence of gravitational acceleration in the crystal growth of bulk electronic materials is in containerless processing. Molten floating zones have been used for many years to prepare single crystals, for which container reaction and contamination are major concerns. However, on Earth, the vertical height of a molten floating zone is limited by the ability of the melt surface tension to counterbalance the hydrostatic pressure effects of gravity. In microgravity, the length of a static molten column is limited only by shape instability criteria. Thus there is greater latitude to manipulate molten floating zones for bulk solidification in microgravity than on Earth.

\section{MAJOR RESEARCH ACCOMPLISHMENTS}

Only the U.S. microgravity research program is reviewed here. So far, the work comprises only a few hundred hours of experimental time, as opposed to much greater experience with ground-based experiments. Also, the use of space for vacuum processing by employing an orbiting molecular wake shield is not considered here, because there are large uncertainties about the gas pressure and dynamics in such an environment and because laboratory conditions on Earth have continued to improve.

The materials selected for study to date have been mostly model systems or have been scaled to small dimensions to accommodate on-orbit power limitations. Thus, when combined with the limited flight experiment opportunities, little new information has been acquired in this area that is directly relevant to the wider set of research priorities in electronic materials. However, the work reported here does contribute to our basic understanding of bulk crystal growth processes, both through theoretical studies of heat and mass transport as well as the experimental study of model systems. The paradigm for these studies is use of the microgravity environment to decouple the interferences of gravitationally driven fluid flows from the effects produced by other forces.

As of January 1991, there were seven ground-based investigations and four flight experiments related to electronic materials in the U.S. microgravity research program. The principal results can be summarized according to the growth method: melt, vapor, or solution growth. 


\section{Melt Growth}

Melt growth experiments have focused on solid solution systems ( $\mathrm{PbSnTe}, \mathrm{HgCdTe})$ in which density gradients due to both temperature and concentration can interact to drive flow. Up to now, experiments have revealed solute segregation and convection phenomena by means of comparison between Earth-based and space-based conditions as well as by comparison with theoretical models. In $\mathrm{Pb}(1-\mathrm{x}) \mathrm{Sn}(\mathrm{x}) \mathrm{Te}$, the rejection of solute on solidification leads to a dynamically unstable density gradient, and complete mixing was observed in the melt under low-gravity conditions. However, acceleration levels were not measured during the growth. Also, the apparatus suffered thermal control problems. In addition, it would have been most useful for the solid-melt interface to have been delineated in order to aid analysis. In $\mathrm{Hg}(1-\mathrm{x}) \mathrm{Cd}(\mathrm{x}) \mathrm{Te}$, for which the rejected solute raises the density and lowers the melting point, no space experiments have been flown; however, extensive ground-based experiments and analysis have demonstrated diffusion-controlled axial segregation for small sample sizes, but large lateral concentration differences. The reasons for this variation are not entirely understood, and low-gravity experiments would give major insight into successful growth conditions for compositional control. For these experiments to be successful, the thermal, compositional, interfacial, and accelerational environments must be controlled and monitored more carefully than they have been in previous experiments.

There has been a trend toward the use of heteroepitaxial growth of solid-solution semiconductor systems on simple elementary or binary compound substrates. For example, $\mathrm{HgCdTe}$ is now grown as epitaxial films on $\mathrm{CdTe}$ substrates. During epitaxial growth, forced convection is used very successfully to improve compositional control, and there is no need to resort to the complex control of bulk melt thermal and solutal parameters; hence, it is unnecessary to use the microgravity environment to study or enhance the crystal growth of these solid solution materials.

\section{Vapor Growth}

Vapor growth of the $\mathrm{HgI}_{2}, \mathrm{GeSe}$, and $\mathrm{HgCdTe}$ systems has been studied in detail.

In $\mathrm{HgI}_{2}$ growth, space experiments have shown that less dislocation motion occurred than in ground-based growth, presumably due to the smaller self-deformation at lower accelerations. The electrical properties of the space-grown crystals improved; both electron and hole mobilities increased by a factor of two, and lifetime increased by a similar factor. However, the results could not be reproduced because these properties change with time 
when the crystals are not properly coated. The enhancement in properties by growth at low accelerations seems real, but the causes of the improvement are not well understood, especially since subsequent mechanical and optical handling are also major factors affecting the performance of this material. Until the measurement and handling protocols are better determined, it does not appear as if more space experiments will lead to more useful information.

In vapor growth of GeSe crystals, space experiments have shown that reduced nucleation and larger crystals can be achieved in closed-tube configurations. Comparison to theory showed that when gas-phase reactions were eliminated, diffusion-limited transport was accomplished in the vapor phase. However, the improved size, surface morphology, and homogeneity of the space-grown crystals are of limited interest since there are no applications for such bulk crystals. Similar experiments with $\mathrm{HgCdTe}$ have been started on the ground by using the information gained from the GeSe system; improvements in both chemical homogeneity and crystalline quality have been realized, and the gas-phase chemistry has been analyzed theoretically. The HgCdTe system would benefit from space experiments yielding results for comparison.

It also has been proposed that epitaxial vapor growth experiments be conducted with the $\mathrm{HgCdTe} / \mathrm{CdTe}$ system. Caution should be exercised here since, as for epitaxial growth from melts, forced convection produces excellent results and it is not clear that low gravity offers any advantage.

\section{Solution Growth}

Solution growth experiments have been conducted for triglycine sulfate (TGS) in space. This material is used for pyroelectric detection of infrared radiation, and the sensitivity of the material is reduced by crystalline imperfections. However, the origins of the loss are not understood. The space experiments were designed to observe fluid flow effects during growth. This experiment was more significant from the point of view of residual fluid motions observed in space than for any improvements in crystalline quality or performance. The experiment is also too complex; the solution is sulfuric acid and must be contained under low-acceleration conditions. Further work in this system has been discontinued because of the small scientific payoffs for the large investments. In essence, aqueous solution growth systems are models for which fluid motion can be directly correlated to crystal growth phenomena. However, there are far better systems that are easier to handle and characterize than TGS; protein crystal growth is one of them. Otherwise, solution growth has no known value to microelectronic materials. 


\section{RESEARCH PROSPECTS AND OPPORTUNITIES}

The results to date are not of widespread interest to the microelectronic research community because the research topics do not address important contemporary issues in microelectronics. Typically, bulk crystals are used as substrates upon which epitaxial films are grown. These epitaxial films are the most widely used material for fabricating microelectronic devices and circuits. There is a weak relationship between the properties of the bulk substrate and the epitaxial film. However, the major research issues relate to control of the atomic layer required in epitaxial film growth. Therefore, bulk crystals, such as those studied in microgravity, are not of primary interest.

Opportunities consist of using model systems to explore the fluid phenomena that occur in crystal growth and that result in measurable changes in crystal composition, microstructure, or electrical properties. These measurements can be compared with theoretical models of the fluid flow and segregation phenomena in order to enhance our fundamental understanding of these basic processes.

However, the choice of the model system should be dictated by scientific principles and not by the expediency of its relationship to any commercial class of materials. Therefore, experiments should be supported and evaluated on the principle of increased understanding of processes. The microgravity program should emphasize processing science and deemphasize categorization of activities by disciplinary areas, such as microelectronics, for which the contributions must be judged against a far more comprehensive scientific base. 
D

\section{Fluids, Interfaces, and Transport}

\section{STATUS}

Fluid and interfacial transport processes are ubiquitous throughout materials and biological systems. Gravitational effects influence these processes through the sedimentation of particles, buoyancy-driven convection, and the distortion of liquid and/or fluid interfaces by hydrostatic pressure. In many cases, gravitational effects limit the operation of materials-processing systems, add complexity, and potentially mask other physicochemical reactions that are under way. Experiments in a microgravity environment provide conditions for unraveling these complex transport processes in many systems. The new fundamental understanding gained from these studies has led to improved system performance on Earth, to new applications of the microgravity laboratory, and to the better operation of fluids-handling systems in space.

These experiments and the supporting analyses of fluid and interfacial processes have played a dominant role in past research programs, making this area one of the best-developed and best-documented fields of microgravity research. Many review papers have appeared on aspects of this subject. The books by Feuerbacher et al. ${ }^{1}$ and Myshkis et al. ${ }^{2}$ are very good references to this material. An extended summary of many of the ideas presented here is available in the report of the discipline working group of NASA that focuses on this research area (Rosenberger ${ }^{3}$ ).

Microgravity research in fluids and interfaces is usually classified either by the fundamental processes under study or by the area of application. Important fundamental processes include (1) diffusive phenomena, (2) body- 
force-driven convection and surface-tension-driven convection in fluids, (3) capillary phenomena, (4) nucleation and growth, (5) solidification and microstructure formation, and (6) flow of multiphase fluids. Applications include transport processes in materials-processing systems, the measurement of thermophysical properties, and fluid and energy management in space.

Classification according to fundamental processes is used here to review the accomplishments and future potential for microgravity research in fluid and interfacial systems. Applications of understanding fundamental transport and interfacial processes to particular systems are examined in the context of the connections between this section and other portions of the report. The discussion here focuses on the current level of understanding of physical phenomena and on the role played by past microgravity research in achieving this understanding. Potential applications of these fundamental results, both on Earth and in space, are highlighted and key issues deserving future research are addressed.

Fundamental studies in transport processes can be divided into two segments: research aimed at the analysis of the effects of coupled transport processes where each process is well understood individually, and research focused on understanding of fluxes of mass, heat, and species in complex systems, where present theory gives a less than adequate description.

Many of the problems in the classical theory of fluid mechanics and interface dynamics fall in the first category, in which mathematical descriptions in terms of conservation laws and constitutive equations are known. Given the availability of a database for the relevant transport coefficients (e.g., thermal conductivities, diffusivities, and fluid viscosities) and the dependence of the coefficients on temperature and concentration, these transport models can be solved by numerical methods to give insight into coupled processes and to design experimental systems. This approach is particularly appealing because of the incredibly high cost of microgravity experiments and the increasing capability of simulation methods to handle ever more complex problems.

There are two major difficulties to the numerical simulation approach. First, the large uncertainty in a typical database for thermophysical properties poses a major limitation. For most technologically interesting materials, these properties are not known with any accuracy and, hence, this uncertainty is transferred to the calculation of the transport processes through the simulation. In fact, many microgravity experimental systems to measure these properties are proposed. Second, the environment of microgravity offers a less symmetric and less predictable laboratory than is typical on Earth. For example, the very constant and unidirectional gravitational acceleration on Earth is replaced in space by a jittering acceleration vector with direction and magnitude that depend on time over a wide range of 
frequencies. The use of symmetry restrictions in the calculations-a common mechanism used by analysts to cut the cost of the simulations-is not justified, and, in many cases, totally predictive simulations must be threedimensional and time-dependent. The difficulties of making these calculations and the uncertainty of thermophysical data dictate continued reliance on low-gravity experimentation.

\section{MAJOR RESEARCH ACCOMPLISHMENTS}

The major research challenges and accomplishments for each of the six areas of interest listed above are described below.

\section{Diffusive Phenomena}

The potential for convection-free experimentation in a microgravity environment was a key motivation for many of the original experiments in space. Myriad experiments, ranging from the solidification of metals and semiconductors to electrophoretic separation of proteins, were performed with the hypothesis that, in microgravity, the absence of bulk fluid motion driven by buoyancy forces on Earth would lead to unique material properties and better separation efficiency. These experiments were partially successful. Electrophoresis experiments in space led to improvement in separation efficiency for model systems. Experiments aimed at achieving diffusion-controlled crystal growth showed the expected behavior for axial solute transport, but displayed the radial nonuniformity that was shown computationally to be characteristic of weak convection. These observations spawned both theoretical studies and Earth-based experiments that have demonstrated the importance of controlling very weak convective flows to obtain highly uniform solute concentrations in microgravity solidification systems.

Removing the influence of convection also has uncovered the importance of secondary mechanisms for diffusive transport. Such mechanisms as the Soret effect for mass transport by a temperature gradient and the complementary Dufour flux for heat transfer driven by a concentration gradient have been shown to be important in many microgravity systems, where the effect would be masked by convection on Earth. Theoretical understanding of these effects in anything but very idealized model systems is severely hampered by the lack of experimental data for transport coefficients.

Experimental measurement of diffusivities and other transport coefficients is an exciting research application of the microgravity environment. Measurement of diffusivities in space is particularly appealing, especially for liquids, because the diffusivity is so low that fluid velocities of less than $1 \mu \mathrm{m} / \mathrm{s}$, hardly avoidable on Earth, can lead to huge errors in the measure- 
ment. Recent measurements of liquid diffusivities in space claim an accuracy of 2 percent.

\section{Convection in Fluids}

More complex transport processes arise when convection is driven by multiple mechanisms. An example is double-diffusive convection, caused by simultaneous temperature and concentration gradients. In many materials-processing systems, these driving forces are affected differently by a low-gravity environment. Other driving forces for convection are independent of gravity and will become dominant in microgravity. Electrodynamic and magnetodynamic forces both fall in this category and have been studied in relation to convection during electrophoresis and to motion induced during magnetic levitation, respectively. Alternatively, magnetic fields have been applied to suppress convection in electrically conducting fluids. In small-scale systems of only a few centimeters on a side, the fields generated by conventional magnetic technology are effective enough to alleviate the need for space experiments. In large experimental systems, magnetic fields used in conjunction with a microgravity experiment might be used to suppress residual convection that would occur otherwise if only microgravity or magnetic fields were used. The possibility of enhancing the microgravity environment through the use of magnetic fields deserves more study.

Perhaps the most important and well-studied form of convection in microgravity is caused by differences in surface tension that arise because of temperature or concentration gradients along a liquid and/or fluid surface-so-called thermocapillary or Marangoni convection. This convection is unavoidable when a clean liquid interface exists in a nonisothermal system and, depending on the geometry of the liquid, can be more intense than motion caused by buoyancy. Moreover, thermocapillary flows show nonlinear transitions to time-periodic and, finally, chaotic convection as the temperature or concentration gradient is increased. Under these conditions, the bulk thermocapillary motion dominates heat and solute transport, and there is little advantage to a space experiment. Although much progress has been made in understanding thermocapillary convection, it is impossible to eliminate it in many systems, and its presence severely limits the possible configurations of low-gravity systems for performing convectionless experiments.

\section{Capillary Phenomena}

Without the hydrostatic pressure of gravity, many fluids systems can exist in space with only surface tension affecting a liquid surface. This class of capillary phenomena includes liquid films, foams, drops, bubbles, and other configurations of liquid and/or fluid surfaces that separate bulk 
phases. Statically levitated liquid drops and suspended bubbles are excellent examples for which fundamentally important experiments are possible only in space. Accordingly, microgravity studies of drop dynamics and coalescence have flourished and have found application as the basis for new measurement methods for liquid viscosity and surface tension. Experimental observations of drop dynamics made early in the NASA microgravity program and later results from Space Shuttle experiments spawned many high-quality, theoretical studies of the fluid mechanics of drops and bubbles and advanced this science considerably.

Because these levitation experiments are containerless (i.e., the droplet does not contact a container wall), these techniques will be very important for measurements at high temperature and of highly reactive materials once experimental systems are available for precise measurement and environmental control.

\section{Nucleation and Growth}

The homogeneous nucleation and growth of a new phase, either liquid or solid, from a parent phase, either gas or liquid, constitute a classical area of physics and chemistry and are generic to many varying technologies, including crystallization from liquid solutions, solidification from undercooled melts, and aerosol formation from the vapor phase. Definitive experiments are extremely difficult on Earth because of the complexities introduced by the need for confining surfaces, which are sites for heterogeneous nucleation, and because of gravitationally induced particle settling. The prolonged availability of a well-controlled microgravity environment will make feasible the first experiments free of these effects. To date, these experiments are limited to either minute samples, which can be levitated effectively using acoustic or magnetic levitation devices, or to very-shortduration experiments in NASA free-fall facilities.

\section{Solidification and Microstructure Formation}

Prediction of the microstructure of a solidifying interface is a key problem in materials processing because of the crucial influence that this structure has on the extrinsic properties of the solid. The dynamic behavior of the solidification interfaces depends on heat and solute transport in the liquid phase as well as on interfacial properties of the melt and/or solid system. In the last decade, the problem of prediction of these microstructures has received intense theoretical attention. The emphasis has been on the search for precise descriptions for the morphogenesis of the microstructure patterns, such as solidification cells and dendrites, that form as a function of changes in macroscopic operating conditions-temperature gradient, 
growth rate, and alloy composition. The formation of these small-scale microstructures during phase change is ubiquitous, occurring not only in crystal growth from melts and solutions, but also in electroplating and phase changes in liquid crystal systems. Significant progress has been made on a number of problems, much of the research having been sponsored by NASA. For example, several recent, intricate theories have appeared to predict the dependence on growth rate and undercooling of the shape of a single dendrite growing in an undercooled melt. Microgravity experiments may be the only means of making distinctions between these theories because convection in the melt has a strong effect on experiments, as has been carefully documented. Similarly, the experimental growth of a rod-like, eutectic material on Earth and in microgravity has demonstrated the influence of convection on microstructure formation. On Earth, only thin-film solidification experiments are relatively free of convection, but these experiments may be altered significantly by the presence of the confining sidewalls.

Theories exist that are beginning to link the nonlinear transitions that describe microstructure evolution during phase change with the flow in the adjacent liquid. These theories have identified new types of interface dynamics, which have not yet been confirmed experimentally.

\section{Flow of Multiphase Fluids}

Multiphase fluids, such as bubbly gases and solid-laden slurries, form the class of perhaps the most industrially important liquids. Not only are many materials processed as multiphase liquids, but gas-liquid systems also appear in heat transfer systems that employ nucleate boiling and in many gas separation technologies. These fluids are not well understood in the sense that constitutive descriptions do not exist that give accurate predictions of the average stress in the multiphase liquid and of segregation of the components in nonhomogeneous flows. The development of these descriptions is one of the outstanding problems in fluid rheology. Low-gravity experiments for isothermal gas-liquid flow will be very important in separating the effect of gas buoyancy for viscous and inertial interactions in the prediction of the rheological behavior of liquids containing many gas bubbles. Studies of mass and heat transfer in two-phase flow will also be important in understanding the effects of microgravity on the operation of classical heat transfer and separation technologies in a space environment.

\section{RESEARCH PROSPECTS AND OPPORTUNITIES}

Further progress in the fundamental understanding of transport processes and interfacial systems in microgravity will rely on the continued growth of theoretical and computational research and on expansion of the experi- 
mental database. The needs are not equal among these components; both the ground-based and microgravity experimental programs are much less developed than theory and should receive special attention. Experiments should aim at probing new phenomena and at clarifying the link between theory and experiment. In these experiments, emphasis should be placed on using well-characterized materials; when the characterization is not complete, appropriate measurements of the properties needed should be sponsored. Because many of the experiments will rely on similar systems, for example, directional solidification and drop levitation, integrated common equipment continues to be essential and should be emphasized, but the equipment should be developed in close collaboration with the principal investigators.

Platforms for these experiments should include short-duration drop tower tests, sounding rocket flights, Shuttle bay experiments, and systems on the Space Station. The microgravity needs for a specific experiment should be considered carefully in order to leverage the most cost-effective environment for the measurements.

Continued development of the ground-based research and analysis program is essential to increasing the vitality of this program. This is particularly true because of the important roles that theory and simulation play in unraveling complex transport processes in microgravity.

\section{REFERENCES}

1. Feuerbacher, N., H. Hamacher, and R.J. Naumann. 1986. Material Science in Space. Springer-Verlag, Berlin.

2. Myshkis, A.D., V.G. Babskii, N.D. Kopachevski, L.A. Slobozhanin, and A.D. Tyuptsov. 1976. Low-Gravity Fluid Mechanics. Springer-Verlag, New York.

3. Rosenberger, F. 1989. Report of the Fluids, Interfaces, and Transport Discipline Working Group. Microgravity Science and Applications Division, National Aeronautics and Space Administration. 
$\mathrm{E}$

\section{Glasses and Ceramics}

\section{STATUS}

Several reports have been published that address the overall field of microgravity research and its various subdisciplines, including glasses and ceramics. In addition to reviewing material prepared by the Glasses and Ceramics Discipline Working Group, the committee examined the most recent pertinent reports. ${ }^{1-4}$

A large part of ceramics and glass research is concerned with controlling the minute flaws that determine the manner in which materials (e.g., pottery, bricks, concrete, glass, and others) fail. Ceramics, held together by strong covalent and ionic bonds, cannot absorb impacts by means of plastic deformation, as do metals. Instead, they crack, often catastrophically. Most of the current research in structural ceramics focuses on toughening mechanisms to help inhibit failure. The essence of ceramic properties is as much in the internal structure of their crystals as in their chemical composition. A ruby and a fire brick may be made from the same substance-aluminum oxide-yet they look and behave quite differently. The key difference lies in how the crystals within the structure are arranged. When ceramic and glass structures are ordered or aligned, they can be a great deal stronger than when crystal boundaries or disorder are present. Because many of the defects leading to the failure of ceramic and glass materials emanate from the surface, processing conditions are important in the quest to improve mechanical performance.

Most advanced ceramics for automobile and aircraft engines, bearings, bio-implants, and microchip wafers involve powder processing and consoli- 
dation techniques. Little benefit is foreseen from the study of traditional solid-state processing/consolidation techniques under microgravity conditions. Avoidance of a container, however, may be an important factor in processing reactive ceramic materials. Harmful effects associated with containers include chemical and structural (e.g., crystal nuclei) contamination of the melt and limitations of the temperature to which the melt can be superheated. Low gravity offers the possibility to perform containerless processing, thus avoiding the contamination and nucleation effects produced by the container. In fact, containerless processing has become somewhat synonymous with ceramic and glass research in space. Containerless processing is an excellent example of how to use low gravity to overcome a problem that has nothing to do with gravity. Containers interfere with what one is trying to accomplish in crystal growth. Chemical reactions at the walls can degrade the quality of a crystal being produced or constrain its shape. Removing the container is the issue, while eliminating gravity is the path to the solution.

Unfortunately, it seems that interest in developing containerless processing techniques has been the driving force for research on ceramics and glasses, rather than the converse. Containerless science, however, includes experimental and theoretical studies of the behavior of melts when removed from the physical contact of confining walls. Such studies include nucleation, glass formation in undercooled states, thermophysical measurements, kinetics of purification, and buoyancy and convection in levitated samples. While it is envisioned that glasses and ceramics might be scrutinized to produce selected benchmark materials that can serve as standards to which future terrestrial technology could aspire, it is highly unrealistic to expect that a technology for either glass or ceramic processing could be deployed economically in space in the foreseeable future.

\section{MAJOR RESEARCH ACCOMPLISHMENTS}

Two experiments on the containerless processing of several different glasses have been flown on the Space Shuttle. These studies examined glass nucleation and crystallization processes and used containerless processing with acoustic positioning to avoid contamination. Both experienced a number of difficulties but did accomplish the melting; several glass crystallization paths, along with bubble behavior and resulting homogenization, were analyzed during cooling. The containerless suspension system did not always keep the sample in place during melting, and fluoride dissociation from a zirconium fluoride melt hindered the predicted ease of optical fiber formation.

Studies of the dynamics of bubbles and droplets, which may lead to the removal of gas-filled bubbles during containerless melting, have captured 
the interest of experts in fluid physics and dynamics. Containerless processing has demonstrated the advantage of allowing for significantly lower critical cooling rates, thus extending the range of materials that can be obtained in glassy form. Early drop tube experiments showed the possibility of glass formation in tantalum oxide systems in which glass formation is difficult. Theories of acoustic behavior in a furnace of particular geometry and theories of bubble behavior have been explored extensively. The fabrication of microballoons and porous foams through the understanding of bubble migration is an example of the common research objectives of initial studies.

\section{RESEARCH PROSPECTS AND OPPORTUNITIES}

Containerless processing of materials from the melt certainly offers the possibility of minimizing contamination from crucible materials, particularly for higher-melting-point glasses and the new oxide superconductors, for example, yttrium barium cuprate. However, similar results can be obtained by variations on the skull melting process, in which the material acts as its own container or is levitated on a flowing gaseous stream. Therefore, containerless melting in the low-gravity environment is not absolutely necessary. New glasses may or may not be possible by containerless melting. This depends on whether heterogeneous nucleation occurs at the free surface and on whether bulk impurities or compositional fluctuations will promote heterogeneous nucleation. A low-gravity environment is advantageous for the study of immiscible liquids. Containerless melting becomes important when one of the phases preferentially wets the container, a situation that seems virtually unavoidable.

The preparation of ultrapure glass fibers for optical communications is a relevant subject for the microgravity program. Most glass fibers today are fabricated from silica-based systems and are prepared by chemical vapor deposition. The chemicals used here are ultrapure so that optical losses due to contaminants can be minimized. However, the silica-based systems are limited in certain parts of the infrared spectrum by fundamental absorption effects. For these wavelengths, other glassy materials (such as halide systems), which do not have chemical sources suitable for vapor deposition, have been proposed. Thus, optical glass fibers must be prepared by pulling them directly from the molten glass precursor. Unfortunately, the need to contain these melts in a normal-gravity environment results in serious contamination. Consequently, containerless processing methods appear to be very attractive for this application. Much preliminary work to screen materials and to explore the relative effects of container contamination and nucleation effects on bulk glasses in novel materials systems can be performed in a reduced-gravity environment. The current microgravity program has 
been developing containerless processing techniques for many years; in fact, the U.S. program is leading European and former Soviet efforts. Therefore, the exploitation of ultrapure glass fibers from new materials systems is worth studying in microgravity.

The preparation of powders by gas-phase reactions in fluidized bed reactors makes study of the kinetics of powder nucleation and growth extremely difficult because of convective currents and particle settling caused by gravity. With only gas diffusion as the major transport process, the nucleation and growth of these particles might be studied more carefully in microgravity by laser light scattering techniques, for example. If the kinetics of flow reactor processes can be understood, then powders of controlled size and size distribution can be designed. In addition, the agglomeration processes of these particles in the gas phase due to particle collisions can be understood. Finally, experiments similar to those described above for gasphase reactions can be performed in aqueous systems. In this case, the attractive van der Waals forces can be overcome by charged particles, by applying the principles of electrical double-layer theory, or by the principles of steric hindrance for organic dispersants. Not only could optimum dispersant concentrations be determined, but fundamental studies of agglomeration could also be carried out over a range of particle sizes far greater than could be achieved in a one-gravity environment.

A low-gravity environment is advantageous for the elimination of thermal and compositional currents that lead to compositional inhomogeneity in crystals and may also permit the fabrication of new composites of constituent materials with differences in density. Further, the effects of studies on Earth-based processes in the low-gravity environment can be greater than any actual use of the low-gravity environment for the processing of glass and ceramic materials. The virtual absence of both desirable and undesirable convective effects in low-gravity processing permits the study of complex processes when convective complications are eliminated.

\section{REFERENCES}

1. Panel on Microgravity Research, Solid State Sciences Committee, Board on Physics and Astronomy. 1986. Microgravity Science and Applications: Report on a Workshop. National Academy Press, Washington, D.C.

2. Review Committee, J. Robert Schrieffer, chairman. 1987. Review of Microgravity Science and Applications Flight Programs-January-March 1987. Universities Space Research Association, Washington, D.C.

3. Space Applications Board. 1988. Industrial Applications of the Microgravity Environment. National Academy Press, Washington, D.C.

4. Aeronautics and Space Engineering Board. 1989. Report of the Committee on a Commercially Developed Space Facility. National Academy Press, Washington, D.C. 


\section{Metals and Alloys}

\section{STATUS}

NASA's current terrestrial microgravity program in metals and alloys involves approximately a dozen investigators working exclusively at universities and national (NASA or government-supported) laboratories. Metals and alloys constitute a critically important category of engineered materials encompassing structural materials, composites, conductors, magnetic materials, solders, brazes, catalysts, and the like. Their economic value is far and away the greatest of any class of materials used in technology. Currently the following areas are receiving attention and support from NASA's Microgravity Science and Applications Division.

\section{Containerless Science}

Containerless science deals with experimental and theoretical studies of competitive nucleation and growth kinetics of undercooled systems, especially in the molten state when removed from physical contact with containers. Among such studies are thermophysical measurements, $x$-ray diffraction, and heat flow modeling to predict and control microstructural development. The techniques and equipment used in the ground-based program include electromagnetic and/or acoustic positioning devices and drop tubes that permit solidification or glass formation at deep undercoolings or by the rapid removal of heat through gas and/or piston quenching. 


\section{Directional Solidification}

The responses of microstructures to thermal gradients, freezing rates, and convection are the topics of directional solidification. Experiments and computer modeling of well-characterized polyphase alloys are performed to demonstrate the interaction of convective flow on solute distribution, phase scaling, interface morphology, and particle and/or bubble migration. Experiments designed to differentiate between theories and to improve the understanding of solidification (e.g., via coupled flow, spiral flow, etc.) are conducted both terrestrially on model transparent systems and in parabolic trajectories on KC-135 aircraft. A well-designed apparatus has been constructed to measure Soret coefficients and diffusion coefficients in order to compare them with computed solutions that are valid in the absence of convection.

\section{Casting, Solidification, and Microstructure}

Understanding how convection and sedimentation affect cast and composite materials is the subject of studies in casting, solidification, and microstructure. Alloy dendritic growth and coarsening behavior in response to a change in gravity vector are major objectives of the program. Computer modeling, which examines thermosolutal convection and microsegregation phenomena in dendritically solidifying alloys (by reducing the magnitude of gravity and changing its direction), is also a major focus. A goal of many ground-based calculations is to assist investigators in designing equipment and experiments for low-gravity environments.

A major area of scientific and technological progress in solidification processing entails the development of quantitative, predictive, microstructural "scaling laws." For dendritic growth under diffusion control, scaling laws predict the growth speed, tip radius, and branch spacing as functions of the material parameters. It is estimated that reducing the acceleration of gravity to 0.001 its Earth value would permit almost an order-of-magnitude gain in the useful range of supersaturation for testing diffusion-controlled dendritic growth. In reduced gravity, not only are dendritic crystals less influenced by convection, but the increased size scale with decreased driving force also enables more accurate morphological measurements.

\section{Thermophysical Properties and Sintering Phenomena}

The measurement of such qualities as surface tension, heat of fusion, heat capacity, and gravitational effects during phase sintering of high-melting-point liquid phases is the objective in studies of thermophysical properties and sintering phenomena. Highly successful ground-based, millisecond-resolution, pulse heating experiments have allowed accurate measurements 
of the thermophysical properties of melts ( $\mathrm{T}>1,500 \mathrm{~K}$ ) for electrically conducting materials. Calculations of surface tension, entropy, and thermal emissivity on supercomputers are essential to interpreting contactless temperature measurements. The theoretical basis for predicting limiting compositions that can be sintered under normal gravity is being established.

\section{MAJOR RESEARCH ACCOMPLISHMENTS}

For numerous and controversial reasons, significant results, mainly from Spacelab and sounding rocket missions, have been infrequent, not reproduced, and few. Results outlined below were obtained primarily from sounding rocket SPAR, Spacelab D-1, and Shuttle missions. Three main areas are addressed: solidification, diffusion coefficients, and modeling of solidification.

\section{Solidification}

The principal refinement of the eutectic spacing for the MnBi-Bi system was observed in microgravity on three separate occasions. This trend in reduced, inter-rod spacing was substantiated in similar terrestrial experiments in which melt convection was dampened by the use of a magnetic field. Several studies showed that alloys prepared in space have morphologies that differ from those of their terrestrial counterparts; for example, the patterns of $\mathrm{Pb} / \mathrm{Tl}$ and $\mathrm{Co}_{2} \mathrm{Sm}_{17} / \mathrm{Co}$ were more regular under microgravity, but distorted by convection on Earth, and the dendritic pattern of an $\mathrm{Al} / \mathrm{Cu}$ space sample showed no radial or longitudinal segregation (indicative of diffusion-controlled growth), higher regularity, a larger size of dendritic array, and a five-times-larger arm spacing. Numerous attempts to prepare finely dispersed monotectic alloys $(\mathrm{Al} / \mathrm{In}, \mathrm{Zn} / \mathrm{Bi})$ by cooling through the miscibility gap showed clearly that, in addition to sedimentation, other phaseseparation mechanisms exist, one of them probably surface-tension-driven migration. Directional solidification of composites $\left(\mathrm{Al}_{2} \mathrm{O}_{3}\right.$ particles in copper) showed that below a critical volume fraction ( $<10$ percent) for which the particles are isolated, a homogeneous distribution could be maintained under microgravity, even for particles larger than $1 \mu \mathrm{m}$.

A clear example of the dominating influence of surface energy effects with respect to microgravity occurs in monotectic alloy systems as the temperature falls below a critical point. Over a range of temperatures below the critical point, one of the liquids exhibits perfect welting behavior so that it essentially encapsulates the other liquid and will coat any container. If final freezing occurs within the temperature range of perfect welting, massive segregation will be produced in the microstructure. Alternatively, if the temperature for the monotectic reaction, which produces a solid and a liquid phase, is below the critical range, a regular well-aligned microstructure is possible by directional solidification. 


\section{Diffusion Coefficients}

Measurements of diffusion coefficients in liquid $\mathrm{Sn}$ in space were obtained with accuracies of better than 0.5 percent ( 10 to 50 times better than under Earth's gravity). Such data, which approach in accuracy those for diffusion in solids, cannot be reached in liquids on Earth because of convective disturbances. Furthermore, it was found that the temperature dependence of the diffusion coefficient is proportional to $\mathrm{T}^{2}$ and does not obey an Arrhenius law. In addition, for the first time evidence was obtained for an isotope effect in a self-diffusion experiment.

The Soret coefficient measured in eutectic $\mathrm{MnBi}-\mathrm{Bi}$ at $100^{\circ} \mathrm{C} / \mathrm{cm}$ indicated that transport in microgravity was comparable to normal diffusion. Solutions of cobalt in the cold ends of the cylindrical samples differed markedly from those in the hot ends-that is, thermotransport (not measurable on Earth because of convection) had taken place-and the concentrations of the isotope $\mathrm{Sn}^{112}$ in the cold and hot ends differed also-that is, under convection-free conditions, thermodiffusion in liquids seems to lead to isotope separation.

\section{Modeling of Solidification}

Great strides have been made in comparing calculated microsegregation and experimental results of vertical Bridgman-Stockbarger microgravity experiments. Obtaining the proper experimental definition of metals-processing operations was shown to require collaboration with both fluids and transport communities. Codes have been produced to simulate nonlinear convection that leads to the formation of microsegregation defects. The consequences of reducing the magnitude of gravity, changing its direction and time dependence on thermosolutal convection, and subsequent segregation effects have been studied in detail. Frequently, theoretical models are based on the assumptions of steady state, axisymmetric flow, and constant heater temperature, whereas computer and analytical models have been developed for the diffusional decay of compositional or doping striations during solidification. Theoretical modeling is playing an important role in designing useful experiments to be performed in space.

\section{RESEARCH PROSPECTS AND OPPORTUNITIES}

\section{General}

The future of NASA's microgravity program lies in the continuation of its Spacelab flight program up to and beyond the commissioning of Space Station Freedom, scheduled for the year 2000. To ensure the success of this ambitious program, an expansion of the ground-based, basic research infra- 
structure should be undertaken at centers of excellence in universities. The conditions necessary for microgravity's practical utilization should be defined, even though this field of experimentation is still in its infancy and subject to delays and the risks of experimental failures in orbit. An alternative experimental program involving an unmanned orbital carrier should be put in place in case the space station system is not realized and to provide other, less expensive alternatives.

\section{Specific}

It is clear from previous experiments in space that microgravity can be viewed as a reference environment in the sense that it eliminates or reduces greatly the influence of one parameter that can play a multiple, but not completely understood, role in many metallurgical experiments. Even quite familiar terrestrial situations transferred to an apparently simple space environment require an extensive development program on Earth with theoretical, numerical, and experimental modeling and an open mind to cope with the unpredictable.

For example, current models of the instability at solid and/or liquid interfaces have demonstrated the complexity of this problem and the various methods of treatment. The simplest models involve 7 parameters, some of them with considerable margins of uncertainty; the most elaborate involve more than 20 such parameters, which does not make comparative analysis easy! By reducing the imprecision resulting from many of these parameters (by means of measurements made under ground-based conditions), the yield of space experiments will be increased, and theoretical modeling will be facilitated.

Any solidification process entails numerous geometrical, thermal, chemical, physical, and hydrodynamic parameters, which must be known if an accurate forecast of instability conditions under both terrestrial and space conditions is to be made. Improvements in the accuracy of some of these parameters are needed and are made possible by taking advantage of the microgravity environment. The improvements derived from such experimental work will benefit other situations of practical interest. Careful, preliminary, ground-based experiments performed concurrently would be worth carrying out since they will make it possible to approach the critical conditions for the onset of interface instability that will prevail in space. By varying the orientation of the growth rate with respect to the gravity vector (i.e., parallel, antiparallel, or perpendicular), one can introduce significant changes in the values of convective flow. Another approach would be to study certain systems enclosed in capillary tubes, which makes it possible to reduce the values of the Rayleigh number, as does reduction of the gravity level. 
In the case of an electrically conducting fluid, a strong damping of convection action can be imposed by using magnetic fields. The effect of such a magnetic field may be to delay the occurrence of convection by increasing the viscosity of the liquid, which introduces a resistance to hydrodynamic instabilities. Organized solute fluxes are then reduced drastically, but the random fluctuations of chemical, and especially of thermal, fluxes are maintained. Additionally, it would be valuable to study, on Earth, systems with minimum values of fractional density change and of thermal and solutal coefficients of expansion, both parameters being the driving forces for convective fluxes.

Freezing a dispersion (gas bubble or particle) with the aim of obtaining a stable material that combines the properties of two different components is desirable. A crucial and theoretically unsolved problem concerns the conditions for incorporation of particles into the advancing solid. Experiments with both immiscible alloys and metal matrix composites would be beneficial. Often, bubbles will be a nuisance in microgravity experiments if they occur unintentionally. The automatic elimination of bubbles by buoyancy does not occur in space. However, the application of a controlled artificial transport creates new problems, usually requiring additional time because the motions that can be induced are slow.

During orbiter accelerations or crew maneuvers, time-dependent variations in acceleration forces ("gravity-jitter") may occur. Their effects on certain phenomena, such as the distribution or redistribution of impurities and/or defects in alloy crystal growth, should be evaluated. Also, because electromagnetic levitation is one of the current techniques employed in containerless processing, it is important to assess quantitatively the role played by residual electromagnetic forces in producing convection and the decay rate of such convection.

Many metals-processing operations involve the simultaneous transfer of matter and thermal energy and the coupling of other transport phenomena. Thermal diffusion (e.g., the Soret effect and the Dufour effect) and electromigration are examples. By carrying out microgravity experiments, the role of convection in modifying these phenomena may be minimized, possibly providing ideal conditions for determining the appropriate transport coefficients for these coupled flux phenomena.

Since 1973, over 1,000 experimental hours have been devoted to microgravity research. These experiments have covered a broad selection of metals and alloys. Many were "firsts" and involved severe restrictions in weight, size of equipment, power, and time; repetition and/or iterations were infrequently allowed. Although microgravity provides a new environment for metals processing, much more fundamental research is needed to use its full potential, as indicated above. The following general fields of metallurgy are recommended for continued microgravity research: 
- Gravity-related aspects of nucleation and growth;

- Accurate measurements of material parameters, for example, thermophysical and diffusion parameters, especially at elevated temperatures;

- Development of microgravity techniques for preparing alloys, for example, containerless processing and skin technology; and

- Preparation of "benchmark" research samples for use as terrestrial standards. 